\title{
Review Article \\ Berezin-Toeplitz Quantization for Compact Kähler Manifolds. A Review of Results
}

\author{
Martin Schlichenmaier \\ Mathematics Research Unit, University of Luxembourg, FSTC, 6, \\ rue Coudenhove-Kalergi 1359 Luxembourg-Kirchberg, Luxembourg \\ Correspondence should be addressed to Martin Schlichenmaier, martin.schlichenmaier@uni.lu
}

Received 30 December 2009; Accepted 5 March 2010

Academic Editor: S. T. Ali

Copyright (C) 2010 Martin Schlichenmaier. This is an open access article distributed under the Creative Commons Attribution License, which permits unrestricted use, distribution, and reproduction in any medium, provided the original work is properly cited.

This article is a review on Berezin-Toeplitz operator and Berezin-Toeplitz deformation quantization for compact quantizable Kähler manifolds. The basic objects, concepts, and results are given. This concerns the correct semiclassical limit behaviour of the operator quantization, the unique BerezinToeplitz deformation quantization (star product), covariant and contravariant Berezin symbols, and Berezin transform. Other related objects and constructions are also discussed.

\section{Introduction}

For quantizable Kähler manifolds the Berezin-Toeplitz (BT) quantization scheme, both the operator quantization and the deformation quantization, supplies canonically defined quantizations. Some time ago, in joint work with Martin Bordemann and Eckhard Meinrenken, the author of this review showed that for compact Kähler manifolds it is a welldefined quantization scheme with correct semiclassical limit [1].

What makes the Berezin-Toeplitz quantization scheme so attractive is that it does not depend on further choices and that it does not only produce a formal deformation quantization, but one which is deeply related to some operator calculus.

From the point of view of classical mechanics, compact Kähler manifolds appear as phase space manifolds of restricted systems or of reduced systems. A typical example of its appearance is given by the spherical pendulum which after reduction has as phase-space the complex projective space.

Very recently, inspired by fruitful applications of the basic techniques of the BerezinToeplitz scheme beyond the quantization of classical systems, the interest in it revived considerably. 
For example, these techniques show up in a noncommutative geometry. More precisely, they appear in the approach to noncommutative geometry using fuzzy manifolds. The quantum spaces of the BT quantization of level $m$, defined in Section 3 further down, are finite-dimensional, and the quantum operator of level $m$ constitutes finite-dimensional noncommutative matrix algebras. This is the arena of noncommutative fuzzy manifolds and gauge theories over them. The classical limit, the commutative manifold, is obtained as limit $m \rightarrow \infty$. The name fuzzy sphere was coined by Madore [2] for a certain quantized version of the Riemann sphere. It turned out to be a quite productive direction in the noncommutative geometry approach to quantum field theory. It is impossible to give a rather complete list of people working in this approach. The following is a rather erratic and random choice of [3-10].

Another appearance of Berezin-Toeplitz quantization techniques as basic ingredients is in the pioneering work of Jørgen Andersen on the mapping class group (MCG) of surfaces in the context of Topological Quantum Field Theory (TQFT). Beside other results, he was able to prove the asymptotic faithfulness of the mapping group action on the space of covariantly constant sections of the Verlinde bundle with respect to the Axelrod-Witten-de la Pietra and Witten connection [11,12]; see also [13]. Furthermore, he showed that the MCG does not have Kazhdan's property $T$. Roughly speaking, a group which has property $T$ says that the identity representation is isolated in the space of all unitary representations of the group [14]. In these applications, the manifolds to be quantized are the moduli spaces of certain flat connections on Riemann surfaces or, equivalently, the moduli space of stable algebraic vector bundles over smooth projective curves. Here further exciting research is going on, in particular, in the realm of TQFT and the construction of modular functors [15-17].

In general, quite often moduli spaces come with a Kähler structure which is quantizable. Hence, it is not surprising that the Berezin-Toeplitz quantization scheme is of importance in moduli space problems. Noncommutative deformations and a quantization being a noncommutative deformation, yield also information about the commutative situation. These aspects clearly need further investigations.

There are a lot of other applications on which work has already been done, recently started, or can be expected. As the Berezin-Toeplitz scheme has become a basic tool, this seems the right time to collect the techniques and results in such a review. We deliberately concentrate on the case of compact Kähler manifolds. In particular, we stress the methods and results valid for all of them. Due to "space-time" limitations, we will not deal with the noncompact situation. In this situation, case by case studies of the examples or class of examples are needed. See Section 3.7 for references to some of them. Also we have to skip presenting recent attempts to deal with special singular situations, like orbifolds, but see at least [18-20].

Of course, there are other reviews presenting similar quantization schemes. A very incomplete list is the following [21-25].

This review is self-contained in the following sense. I try to explain all notions and concepts needed to understand the results and theorems only assuming some background in modern geometry and analysis. And as such it should be accessible for a newcomer to the field (both for mathematicians as for physicists) and help him to enter these interesting research directions. It is not self-contained in the strict sense as it does supply only those proofs or sketches of proofs which are either not available elsewhere or are essential for the understanding of the statements and concepts. The review does not require a background in quantum physics as only mathematical aspects of quantizations are touched on. 


\section{The Set-Up of Geometric Quantization}

In the following, I will recall the principal set-up of geometric quantization which is usually done for symplectic manifolds in the case when the manifold is a Kähler manifold.

\subsection{Kähler Manifolds}

We will only consider phase-space manifolds which carry the structure of a Kähler manifold $(M, \omega)$. Recall that in this case $M$ is a complex manifold and $\omega$, the Kähler form, is a nondegenerate closed positive $(1,1)$-form.

If the complex dimension of $M$ is $n$, then the Kähler form $\omega$ can be written with respect to local holomorphic coordinates $\left\{z_{i}\right\}_{i=1, \ldots, n}$ as

$$
\omega=\mathrm{i} \sum_{i, j=1}^{n} g_{i j}(z) d z_{i} \wedge d \bar{z}_{j}
$$

with local functions $g_{i j}(z)$ such that the matrix $\left(g_{i j}(z)\right)_{i, j=1, \ldots, n}$ is hermitian and positive definite.

Later on we will assume that $M$ is a compact Kähler manifold.

\subsection{Poisson Algebra}

Denote by $C^{\infty}(M)$ the algebra of complex-valued (arbitrary often) differentiable functions with the point-wise multiplication as an associative product. A symplectic form on a differentiable manifold is a closed nondegenerate 2-form. In particular, we can consider our Kähler form $\omega$ as a symplectic form.

For symplectic manifolds, we can introduce on $C^{\infty}(M)$ a Lie algebra structure, the Poisson bracket Poisson bracket $\{\cdot \cdot \cdot \cdot$, in the following way. First we a assign to every $f \in$ $C^{\infty}(M)$ its Hamiltonian vector field $X_{f}$, and then to every pair of functions $f$ and $g$ the Poisson bracket $\{\cdot, \cdot\}$ via

$$
\omega\left(X_{f}, \cdot\right)=d f(\cdot), \quad\{f, g\}:=\omega\left(X_{f}, X_{g}\right)
$$

One verifies that this defines a Lie algebra structures and that furthermore, we have the Leibniz rule

$$
\{f g, h\}=f\{g, h\}+\{f, h\} g, \quad \forall f, g, h \in C^{\infty}(M) .
$$

Such a compatible structure is called a Poisson algebra. 


\subsection{Quantum Line Bundles}

A quantum line bundle for a given symplectic manifold $(M, \omega)$ is a triple $(L, h, \nabla)$, where $L$ is a complex line bundle, $h$ a Hermitian metric on $L$, and $\nabla$ a connection compatible with the metric $h$ such that the (pre)quantum condition

$$
\begin{aligned}
\operatorname{curv}_{L, \nabla}(X, Y):= & \nabla_{X} \nabla_{Y}-\nabla_{Y} \nabla_{X}-\nabla_{[X, Y]}=-i \omega(X, Y) \\
& \text { resp., } \quad \operatorname{curv}_{L, \nabla}=-i \omega
\end{aligned}
$$

is fulfilled. A symplectic manifold is called quantizable if there exists a quantum line bundle.

In the situation of Kähler manifolds, we require for a quantum line bundle to be holomorphic and that the connection is compatible both with the metric $h$ and the complex structure of the bundle. In fact, by this requirement $\nabla$ will be uniquely fixed. If we choose local holomorphic coordinates on the manifold and a local holomorphic frame of the bundle, the metric $h$ will be represented by a function $\widehat{h}$. In this case, the curvature in the bundle can be given by $\bar{\partial} \partial \log \widehat{h}$ and the quantum condition reads as

$$
\mathrm{i} \bar{\partial} \partial \log \widehat{h}=\omega
$$

\subsection{Example: The Riemann Sphere}

The Riemann sphere is the complex projective line $\mathbb{P}^{1}(\mathbb{C})=\mathbb{C} \cup\{\infty\} \cong S^{2}$. With respect to the quasiglobal coordinate $z$, the form can be given as

$$
\omega=\frac{\mathrm{i}}{(1+z \bar{z})^{2}} d z \wedge d \bar{z}
$$

For the Poisson bracket, one obtains

$$
\{f, g\}=\mathrm{i}(1+z \bar{z})^{2}\left(\frac{\partial f}{\partial \bar{z}} \cdot \frac{\partial g}{\partial z}-\frac{\partial f}{\partial z} \frac{\partial g}{\partial \bar{z}}\right)
$$

Recall that the points in $\mathbb{P}^{1}(\mathbb{C})$ correspond to lines in $\mathbb{C}^{2}$ passing through the origin. If we assign to every point in $\mathbb{P}^{1}(\mathbb{C})$ the line it represents, we obtain a holomorphic line bundle, called the tautological line bundle. The hyper plane section bundle is dual to the tautological bundle. It turns out that it is a quantum line bundle. Hence $\mathbb{P}^{1}(\mathbb{C})$ is quantizable. 


\subsection{Example: The Complex Projective Space}

Next we consider the $n$-dimensional complex projective space $\mathbb{P}^{n}(\mathbb{C})$. The example above can be extended to the projective space of any dimension. The Kähler form is given by the FubiniStudy form

$$
\omega_{\mathrm{FS}}:=\mathrm{i} \frac{\left(1+|w|^{2}\right) \sum_{i=1}^{n} d w_{i} \wedge d \bar{w}_{i}-\sum_{i, j=1}^{n} \bar{w}_{i} w_{j} d w_{i} \wedge d \bar{w}_{j}}{\left(1+|w|^{2}\right)^{2}}
$$

The coordinates $w_{j}, j=1, \ldots, n$, are affine coordinates $w_{j}=z_{j} / z_{0}$ on the affine chart $U_{0}:=$ $\left\{\left(z_{0}: z_{1}: \cdots: z_{n}\right) \mid z_{0} \neq 0\right\}$. Again, $\mathbb{P}^{n}(\mathbb{C})$ is quantizable with the hyper plane section bundle as a quantum line bundle.

\subsection{Example: The Torus}

The (complex-) one-dimensional torus can be given as $M=\mathbb{C} / \Gamma_{\tau}$, where $\Gamma_{\tau}:=\{n+m \tau \mid$ $n, m \in \mathbb{Z}\}$ is a lattice with $\operatorname{Im} \tau>0$. As Kähler form, we take

$$
\omega=\frac{\mathrm{i} \pi}{\operatorname{Im} \tau} d z \wedge d \bar{z}
$$

with respect to the coordinate $z$ on the covering space $\mathbb{C}$. Clearly this form is invariant under the lattice $\Gamma_{\tau}$ and hence well defined on $M$. For the Poisson bracket, one obtains

$$
\{f, g\}=\mathrm{i} \frac{\operatorname{Im} \tau}{\pi}\left(\frac{\partial f}{\partial \bar{z}} \cdot \frac{\partial g}{\partial z}-\frac{\partial f}{\partial z} \frac{\partial g}{\partial \bar{z}}\right)
$$

The corresponding quantum line bundle is the theta line bundle of degree 1 , that is, the bundle whose global sections are scalar multiples of the Riemann theta function.

\subsection{Example: The Unit Disc and Riemann Surfaces}

The unit disc

$$
\Phi:=\{z \in \mathbb{C}|| z \mid<1\}
$$

is a noncompact Kähler manifold. The Kähler form is given by

$$
\omega=\frac{2 i}{(1-z \bar{z})^{2}} d z \wedge d \bar{z} .
$$


Every compact Riemann surface $M$ of genus $g \geq 2$ can be given as the quotient of the unit disc under the fractional linear transformations of a Fuchsian subgroup of $S U(1,1)$. If $R=$ $((a / b)(b / a))$ with $|a|^{2}-|b|^{2}=1$ (as an element of $\left.S U(1,1)\right)$, then the action is

$$
z \longmapsto R(z):=\frac{a z+b}{\bar{b} z+\bar{a}}
$$

The Kähler form (2.12) is invariant under the fractional linear transformations. Hence it defines a Kähler form on $M$. The quantum bundle is the canonical bundle, that is, the bundle whose local sections are the holomorphic differentials. Its global sections can be identified with the automorphic forms of weight 2 with respect to the Fuchsian group.

\subsection{Consequences of Quantizability}

The above examples might create the wrong impression that every Kähler manifold is quantizable. This is not the case. For example, only those higher-dimensional tori complex tori are quantizable which are abelian varieties, that is, which admit enough theta functions. It is well known that for $n \geq 2$ a generic torus will not be an abelian variety. Why this implies that they will not be quantizable, we will see in a moment.

In the language of differential geometry, a line bundle is called a positive line bundle if its curvature form (up to a factor of $1 / \mathrm{i}$ ) is a positive form. As the Kähler form is positive, the quantum condition (2.4) yields that a quantum line bundle $L$ is a positive line bundle.

\subsection{Embedding into Projective Space}

In the following, we assume that $M$ is a quantizable compact Kähler manifold with quantum line bundle $L$. Kodaira's embedding theorem says that $L$ is ample, that is, that there exists a certain tensor power $L^{m_{0}}$ of $L$ such that the global holomorphic sections of $L^{m_{0}}$ can be used to embed the phase space manifold $M$ into the projective space of suitable dimension. The embedding is defined as follows. Let $\Gamma_{\text {hol }}\left(M, L^{m_{0}}\right)$ be the vector space of global holomorphic sections of the bundle $L^{m_{0}}$. Fix a basis $s_{0}, s_{1}, \ldots, s_{N}$. We choose local holomorphic coordinates $z$ for $M$ and a local holomorphic frame $e(z)$ for the bundle $L$. After these choices, the basis elements can be uniquely described by local holomorphic functions $\widehat{s}_{0}, \widehat{s}_{1}, \ldots, \widehat{s}_{N}$ defined via $s_{j}(z)=\widehat{s}_{j}(z) e(z)$. The embedding is given by the map

$$
M \hookrightarrow \mathbb{P}^{N}(\mathbb{C}), \quad z \longmapsto \phi(z)=\left(\widehat{s}_{0}(z): \widehat{s}_{1}(z): \cdots: \widehat{s}_{N}(z)\right)
$$

Note that the point $\phi(z)$ in projective space neither depends on the choice of local coordinates nor on the choice of the local frame for the bundle $L$. Furthermore, a different choice of basis corresponds to a $\operatorname{PGL}(N, \mathbb{C})$ action on the embedding space and hence the embeddings are projectively equivalent.

By this embedding, quantizable compact Kähler manifolds are complex submanifolds of projective spaces. By Chow's theorem [26], they can be given as zero sets of homogenous polynomials, that is, they are smooth projective varieties. The converse is also true. Given 
a smooth subvariety $M$ of $\mathbb{P}^{n}(\mathbb{C})$, it will become a Kähler manifold by restricting the FubiniStudy form. The restriction of the hyper plane section bundle will be an associated quantum line bundle.

At this place a warning is necessary. The embedding is only an embedding as complex manifolds are not an isometric embedding as Kähler manifolds. This means that in general $\phi^{-1}\left(\omega_{\mathrm{FS}}\right) \neq \omega$. See Section 7.6 for results on an "asymptotic expansion" of the pullback.

A line bundle, whose global holomorphic sections will define an embedding into projective space, is called a very ample line bundle. In the following, we will assume that $L$ is already very ample. If $L$ is not very ample, we choose $m_{0} \in \mathbb{N}$ such that the bundle $L^{m_{0}}$ is very ample and take this bundle as quantum line bundle with respect to the rescaled Kähler form $m_{0} \omega$ on $M$. The underlying complex manifold structure will not change.

\section{Berezin-Toeplitz Operators}

In this section, we will consider an operator quantization. This says that we will assign to each differentiable (differentiable will always mean differentiable to any order) function $f$ on our Kähler manifold $M$ (i.e., on our "phase space") the Berezin-Toeplitz (BT) quantum operator $T_{f}$. More precisely, we will consider a whole family of operators $T_{f}^{(m)}$. These operators are defined in a canonical way. As we know from the Groenewold-van Howe theorem, we cannot expect that the Poisson bracket on $M$ can be represented by the Lie algebra of operators if we require certain desirable conditions see [27] for further details. The best we can expect is that we obtain it at least "asymptotically". In fact, this is true.

In our context also the operator of geometric quantization exists. At the end of this section, we will discuss its relation to the BT quantum operator. It will turn out that if we take for the geometric quantization the Kähler polarization then they have the same asymptotic behaviour.

\subsection{Tensor Powers of the Quantum Line Bundle}

Let $(M, \omega)$ be a compact quantizable Kähler manifold and $(L, h, \nabla)$ a quantum line bundle. We assume that $L$ is already very ample. We consider all its tensor powers

$$
\left(L^{m}, h^{(m)}, \nabla^{(m)}\right)
$$

Here $L^{m}:=L^{\otimes m}$. If $\widehat{h}$ corresponds to the metric $h$ with respect to a local holomorphic frame $e$ of the bundle $L$, then $\widehat{h}^{m}$ corresponds to the metric $h^{(m)}$ with respect to the frame $e^{\otimes m}$ for the bundle $L^{m}$. The connection $\nabla^{(m)}$ will be the induced connection.

We introduce a scalar product on the space of sections. In this review, we adopt the convention that a hermitian metric (and a scalar product) is antilinear in the first argument and linear in the second argument. First we take the Liouville form $\Omega=(1 / n !) \omega^{\wedge n}$ as a volume form on $M$ and then set for the scalar product and the norm

$$
\langle\varphi, \psi\rangle:=\int_{M} h^{(m)}(\varphi, \psi) \Omega, \quad\|\varphi\|:=\sqrt{\langle\varphi, \varphi\rangle},
$$


on the space $\Gamma_{\infty}\left(M, L^{m}\right)$ of global $C^{\infty}$-sections. Let $L^{2}\left(M, L^{m}\right)$ be the $L^{2}$-completion of $\Gamma_{\infty}\left(M, L^{m}\right)$, and $\Gamma_{\text {hol }}\left(M, L^{m}\right)$ its (due to the compactness of $M$ ) finite-dimensional closed subspace of global holomorphic sections. Let

$$
\Pi^{(m)}: \mathrm{L}^{2}\left(M, L^{m}\right) \longrightarrow \Gamma_{\text {hol }}\left(M, L^{m}\right)
$$

be the projection onto this subspace.

Definition 3.1. For $f \in C^{\infty}(M)$, the Toeplitz operator $T_{f}^{(m)}$ (of level $m$ ) is defined by

$$
T_{f}^{(m)}:=\Pi^{(m)}(f \cdot): \Gamma_{\mathrm{hol}}\left(M, L^{m}\right) \longrightarrow \Gamma_{\mathrm{hol}}\left(M, L^{m}\right) .
$$

In words, one takes a holomorphic section $s$ and multiplies it with the differentiable function $f$. The resulting section $f \cdot s$ will only be differentiable. To obtain a holomorphic section, one has to project it back on the subspace of holomorphic sections.

The linear map

$$
T^{(m)}: C^{\infty}(M) \longrightarrow \operatorname{End}\left(\Gamma_{\mathrm{hol}}\left(M, L^{m}\right)\right), \quad f \longrightarrow T_{f}^{(m)}=\Pi^{(m)}(f \cdot), \quad m \in \mathbb{N}_{0}
$$

is the Toeplitz or Berezin-Toeplitz quantization map (of level $m$ ). It will neither be a Lie algebra homomorphism nor an associative algebra homomorphism as in general

$$
T_{f}^{(m)} T_{g}^{(m)}=\Pi^{(m)}(f \cdot) \Pi^{(m)}(g \cdot) \Pi^{(m)} \neq \Pi^{(m)}(f g \cdot) \Pi=T_{f g}^{(m)} .
$$

Furthermore, on a fixed level $m$, it is a map from the infinite-dimensional commutative algebra of functions to a noncommutative finite-dimensional (matrix) algebra. The finitedimensionality is due to the compactness of $M$. A lot of classical information will get lost. To recover this information, one has to consider not just a single level $m$ but all levels together.

Definition 3.2. The Berezin-Toeplitz quantization is the map

$$
C^{\infty}(M) \longrightarrow \prod_{m \in \mathbb{N}_{0}} \operatorname{End}\left(\Gamma_{\text {hol }}\left(M, L^{(m)}\right)\right), \quad f \longrightarrow\left(T_{f}^{(m)}\right)_{m \in \mathbb{N}_{0}} .
$$

We obtain a family of finite-dimensional (matrix) algebras and a family of maps. This infinite family should in some sense "approximate" the algebra $C^{\infty}(M)$.

\subsection{Approximation Results}

Denote for $f \in C^{\infty}(M)$ by $|f|_{\infty}$, the supnorm of $f$ on $M$ and by

$$
\left\|T_{f}^{(m)}\right\|:=\sup _{\substack{s \in \Gamma_{\text {hol }}\left(M, L^{m}\right) \\ s \neq 0}} \frac{\left\|T_{f}^{(m)} s\right\|}{\|s\|},
$$


the operator norm with respect to the norm (3.2) on $\Gamma_{\text {hol }}\left(M, L^{m}\right)$. The following theorem was shown in 1994.

Theorem 3.3 (Bordemann et al. [1]). (a) For every $f \in C^{\infty}(M)$, there exists a $C>0$ such that

$$
|f|_{\infty}-\frac{C}{m} \leq\left\|T_{f}^{(m)}\right\| \leq|f|_{\infty}
$$

In particular, $\lim _{m \rightarrow \infty}\left\|T_{f}^{(m)}\right\|=|f|_{\infty}$.

(b) For every $f, g \in C^{\infty}(M)$,

$$
\left\|m \mathrm{i}\left[T_{f}^{(m)}, T_{g}^{(m)}\right]-T_{\{f, g\}}^{(m)}\right\|=O\left(\frac{1}{m}\right) .
$$

(c) For every $f, g \in C^{\infty}(M)$,

$$
\left\|T_{f}^{(m)} T_{g}^{(m)}-T_{f \cdot g}^{(m)}\right\|=O\left(\frac{1}{m}\right) .
$$

These results are contained in Theorems 4.1, 4.2, and in Section 5 in [1]. We will indicate the proof for (b) and (c) in Section 5. It will make reference to the symbol calculus of generalised Toeplitz operators as developed by Boutet de Monvel and Guillemin [28]. The original proof of (a) was quite involved and required Hermite distributions and related objects. On the basis of the asymptotic expansion of the Berezin transform [29], a more direct proof can be given. I will discuss this in Section 7.3.

Only on the basis of this theorem, we are allowed to call our scheme a quantizing scheme. The properties in the theorem might be rephrased as the BT operator quantization has the correct semiclassical limit.

\subsection{Further Properties}

From Theorem 3.3 (c), we have the following proposition.

Proposition 3.4. Let $f_{1}, f_{2}, \ldots, f_{r} \in C^{\infty}(M)$; then

$$
\left\|T_{f_{1} \ldots f_{r}}^{(m)}-T_{f_{1}}^{(m)} \cdots T_{f_{r}}^{(m)}\right\|=O\left(m^{-1}\right)
$$

follows directly.

\section{Proposition 3.5.}

$$
\lim _{m \rightarrow \infty}\left\|\left[T_{f}^{(m)}, T_{g}^{(m)}\right]\right\|=0 .
$$


Proof. Using the left side of the triangle inequality, from Theorem 3.3 (b), it follows that

$$
\left|m\left\|\left[T_{f}^{(m)}, T_{g}^{(m)}\right]\right\|-\left\|T_{\{f, g\}}^{(m)}\right\|\right| \leq\left\|m \mathrm{i}\left[T_{f}^{(m)}, T_{g}^{(m)}\right]-T_{\{f, g\}}^{(m)}\right\|=O\left(\frac{1}{m}\right) .
$$

By part (a) of the theorem $\left\|T_{\{f, g\}}^{(m)}\right\| \rightarrow|\{f, g\}|_{\infty}$, and it stays finite. Hence $\left\|\left[T_{f}^{(m)}, T_{g}^{(m)}\right]\right\|$ has to be a zero sequence.

Proposition 3.6. The Toeplitz map

$$
C^{\infty}(M) \longrightarrow \operatorname{End}\left(\Gamma_{\text {hol }}\left(M, L^{(m)}\right)\right), \quad f \longrightarrow T_{f}^{(m)},
$$

is surjective.

For a proof, see [1, Proposition 4.2].

This proposition says that for a fixed $m$ every operator $A \in \operatorname{End}\left(\Gamma_{\text {hol }}\left(M, L^{(m)}\right)\right)$ is the Toeplitz operator of a function $f_{m}$. In the language of Berezin's co- and contravariant symbols, $f_{m}$ will be the contravariant symbol of $A$. We will discuss this in Section 6.2.

Proposition 3.7. For all $f \in C^{\infty}(M)$,

$$
T_{f}^{(m)^{*}}=T_{\bar{f}}^{(m)}
$$

In particular, for real valued functions $f$ the associated Toeplitz operator is self-adjoint.

Proof. Take $s, t \in \Gamma_{\text {hol }}\left(M, L^{m}\right)$; then

$$
\left\langle s, T_{f}^{(m)} t\right\rangle=\left\langle s, \Pi^{(m)}(f \cdot t)\right\rangle=\langle s, f \cdot t\rangle=\langle\bar{f} \cdot s, t\rangle=\left\langle T_{\bar{f}}^{(m)} s, t\right\rangle .
$$

The opposite of the last statement of the above proposition is also true in the following sense.

Proposition 3.8. Let $A \in \operatorname{End}\left(\Gamma_{\mathrm{hol}}\left(M, L^{(m)}\right)\right)$ be a self-adjoint operator; then there exists a real valued function $f$, such that $A=T_{f}^{(m)}$.

Proof. By the surjectivity of the Toeplitz map $A=T_{f}^{(m)}$ with a complex-valued function $f=$ $f_{0}+\mathrm{i} f_{1}$ with real functions $f_{0}$ and $f_{1}$. As $T_{f}^{(m)}=A=A^{*}=T_{\bar{f}}^{(m)}$, it follows that $T_{f-\bar{f}}=0$ and hence $T_{f_{1}}^{(m)}=0$. From this we conclude that $A=T_{f}^{(m)}=T_{f_{1}}^{(m)}$.

We like to stress the fact that the Toeplitz map is never injective on a fixed level $\mathrm{m}$. Only if $\left\|T_{f-g}^{(m)}\right\| \rightarrow 0$ for $m \rightarrow 0$, we can conclude that $f=g$. 
Proposition 3.9. Let $f \in C^{\infty}(M)$ and $n=\operatorname{dim}_{\mathbb{C}} M$. Denote the trace on $\operatorname{End}\left(\Gamma_{\text {hol }}\left(M, L^{m}\right)\right)$ by $\operatorname{Tr}^{(m)}$, then

$$
\operatorname{Tr}^{(m)}\left(T_{f}^{(m)}\right)=m^{n}\left(\frac{1}{\operatorname{vol}\left(\mathbb{P}^{n}(\mathbb{C})\right)} \int_{M} f \Omega+O\left(m^{-1}\right)\right)
$$

See [1], respectively [30] for a detailed proof.

\subsection{Strict Quantization}

The asymptotic results of Theorem 3.3 say that the BT operator quantization is a strict quantization in the sense of Rieffel [31] as formulated in the book by Landsman [32]. We take as base space $X=\{0\} \cup\{1 / m \mid m \in \mathbb{N}\}$, with its induced topology coming from $\mathbb{R}$. Note that $\{0\}$ is an accumulation point of the set $\{1 / m \mid m \in \mathbb{N}\}$. As $C^{*}$ algebras above the points $\{1 / m\}$, we take the algebras $\operatorname{End}\left(\Gamma_{\text {hol }}\left(M, L^{(m)}\right)\right)$ and above $\{0\}$ the algebra $C^{\infty}(M)$. For $f \in C^{\infty}(M)$, we assign $0 \mapsto f$ and $1 / m \mapsto T_{f}^{(m)}$. Now the property (a) in Theorem 3.3 is called in [32] Rieffel's condition, (b) Dirac's condition, and (c) von Neumann's condition. Completeness is true by Propositions 3.6 and 3.8.

This definition is closely related to the notion of continuous fields of $C^{*}$-algebras; see [32].

\subsection{Relation to Geometric Quantization}

There exists another quantum operator in the geometric setting, the operator of geometric quantization introduced by Kostant and Souriau. In a first step, the prequantum operator associated to the bundle $L^{m}$ for the function $f \in C^{\infty}(M)$ is defined as

$$
P_{f}^{(m)}:=\nabla_{X_{f}^{(m)}}^{(m)}+\mathrm{i} f \cdot i d
$$

Here $\nabla^{(m)}$ is the connection in $L^{m}$, and $X_{f}^{(m)}$ the Hamiltonian vector field of $f$ with respect to the Kähler form $\omega^{(m)}=m \cdot \omega$, that is, $m \omega\left(X_{f}^{(m)}, \cdot\right)=d f(\cdot)$. This operator $P_{f}^{(m)}$ acts on the space of differentiable global sections of the line bundle $L^{m}$. The sections depend at every point on $2 n$ local coordinates and one has to restrict the space to sections covariantly constant along the excessive dimensions. In technical terms, one chooses a polarization. In general such a polarization is not unique. But in our complex situation, there is a canonical one by only taking the holomorphic sections. This polarization is called Kähler polarization. The operator of geometric quantization is then defined by the following proposition.

$$
Q_{f}^{(m)}:=\Pi^{(m)} P_{f}^{(m)}
$$

The Toeplitz operator and the operator of geometric quantization (with respect to the Kähler polarization) are related by the following. 
Proposition 3.10 (Tuynman Lemma). Let $M$ be a compact quantizable Kähler manifold; then

$$
Q_{f}^{(m)}=\mathrm{i} \cdot T_{f-(1 / 2 m) \Delta f^{\prime}}^{(m)}
$$

where $\Delta$ is the Laplacian with respect to the Kähler metric given by $\omega$.

For the proof, see $[33,34]$ for a coordinate independent proof.

In particular, the $Q_{f}^{(m)}$ and the $T_{f}^{(m)}$ have the same asymptotic behaviour. We obtain for $Q_{f}^{(m)}$ similar results as in Theorem 3.3. For details see [35]. It should be noted that for (3.21) the compactness of $M$ is essential.

\section{6. $L_{\alpha}$ Approximation}

In [34] the notion of $L_{\alpha}$, respectively, $\mathrm{gl}(N)$, respectively, $\mathrm{su}(N)$ quasilimit were introduced. It was conjectured in [34] that for every compact quantizable Kähler manifold, the Poisson algebra of functions is a $\mathrm{gl}(N)$ quasilimit. In fact, the conjecture follows from Theorem 3.3; see $[1,35]$ for details.

\subsection{The Noncompact Situation}

Berezin-Toeplitz operators can be introduced for noncompact Kähler manifolds. In this case the $L^{2}$ spaces are the space of bounded sections and for the subspaces of holomorphic sections one can only consider the bounded holomorphic sections. Unfortunately, in this context the proofs of Theorem 3.3 do not work. One has to study examples or classes of examples case by case in order to see whether the corresponding properties are correct.

In the following, we give a very incomplete list of references. Berezin himself studied bounded complex-symmetric domains [36]. In this case the manifold is an open domain in $\mathbb{C}^{n}$. Instead of sections one studies functions which are integrable with respect to a suitable measure depending on $\hbar$. Then $1 / \hbar$ corresponds to the tensor power of our bundle. Such Toeplitz operators were studied extensively by Upmeier in a series of works [37-40]. See also the book of Upmeier [41]. For $\mathbb{C}^{n}$ see Berger and Coburn [42, 43]. Klimek and Lesniewski $[44,45]$ studied the Berezin-Toeplitz quantization on the unit disc. Using automorphic forms and the universal covering, they obtain results for Riemann surfaces of genus $g \geq 2$. The names of Borthwick, Klimek, Lesniewski, Rinaldi, and Upmeier should be mentioned in the context of BT quantization for Cartan domains and super Hermitian spaces.

A quite different approach to Berezin-Toeplitz quantization is based on the asymptotic expansion of the Bergman kernel outside the diagonal. This was also used by the author together with Karabegov [29] for the compact Kähler case. See Section 7 for some details. Engliš [46] showed similar results for bounded pseudoconvex domains in $\mathbb{C}^{N}$. Ma and Marinescu [18, 19] developed a theory of Bergman kernels for the symplectic case, which yields also results on the Berezin-Toeplitz operators for certain noncompact Kähler manifolds and even orbifolds. 


\section{Berezin-Toeplitz Deformation Quantization}

There is another approach to quantization. Instead of assigning noncommutative operators to commuting functions, one might think about "deforming" the pointwise commutative multiplication of functions into a noncommutative product. It is required to remain associative, the commutator of two elements should relate to the Poisson bracket of the elements, and it should reduce in the "classical limit" to the commutative situation.

It turns out that such a deformation which is valid for all differentiable functions cannot exist. A way out is to enlarge the algebra of functions by considering formal power series over them and to deform the product inside this bigger algebra. A first systematic treatment and applications in physics of this idea were given in 1978 by Bayen et al. [47, 48]. There the notion of deformation quantization and star products were introduced. Earlier versions of these concepts were around due to Berezin [49], Moyal [50], and Weyl [51]. For a presentation of the history, see [24].

We will show that for compact Kähler manifolds $M$, there is a natural star product.

\subsection{Definition of Star Products}

We start with a Poisson manifold $(M,\{\cdot, \cdot\})$, that is, a differentiable manifold with a Poisson bracket for the function such that $\left(C^{\infty}(M), \cdot,\{\cdot, \cdot\}\right)$ is a Poisson algebra. Let $\mathcal{A}=C^{\infty}(M)[[v]]$ be the algebra of formal power series in the variable $v$ over the algebra $C^{\infty}(M)$.

Definition 4.1. A product $\star$ on $A$ is called a (formal) star product for $M$ (or for $C^{\infty}(M)$ ) if it is an associative $\mathbb{C}[[\mathcal{v}]]$-linear product which is $\mathcal{v}$-adically continuous such that

(1) $A / v A \cong C^{\infty}(M)$, that is, $f \star g \bmod v=f \cdot g$,

(2) $(1 / v)(f \star g-g \star f) \bmod v=-\mathrm{i}\{f, g\}$,

where $f, g \in C^{\infty}(M)$.

Alternatively, we can write

$$
f \star g=\sum_{j=0}^{\infty} C_{j}(f, g) v^{j},
$$

with $C_{j}(f, g) \in C^{\infty}(M)$ such that the $C_{j}$ are bilinear in the entries $f$ and $g$. The conditions (1) and (2) can be reformulated as

$$
C_{0}(f, g)=f \cdot g, \quad C_{1}(f, g)-C_{1}(g, f)=-\mathrm{i}\{f, g\}
$$

By the $v$-adic continuity, (4.1) fixes $\star$ on $\mathcal{A}$. A (formal) deformation quantization is given by a (formal) star product. I will use both terms interchangeable.

There are certain additional conditions for a star product which are sometimes useful.

(1) We call it "null on constants" if $1 \star f=f \star 1=f$, which is equivalent to the fact that the constant function 1 will remain the unit in $\mathcal{A}$. In terms of the coefficients, it can be formulated as $C_{k}(f, 1)=C_{k}(1, f)=0$ for $k \geq 1$. In this review, we always assume this to be the case for star products. 
(2) We call it self-adjoint if $\overline{f \star g}=\bar{g} \star \bar{f}$, where we assume $\bar{v}=v$.

(3) We call it local if

$$
\operatorname{supp} C_{j}(f, g) \subseteq \operatorname{supp} f \cap \operatorname{supp} g, \quad \forall f, g \in C^{\infty}(M)
$$

From the locality property, it follows that the $C_{j}$ are bidifferential operators and that the global star product defines for every open subset $U$ of $M$ a star product for the Poisson algebra $C^{\infty}(U)$. Such local star products are also called differential star products.

\subsection{Existence of Star Products}

In the usual setting of deformation theory, there always exists a trivial deformation. This is not the case here, as the trivial deformation of $C^{\infty}(M)$ to $\mathcal{A}$, which is nothing else as extending the point-wise product to the power series, is not allowed as it does not fulfil Condition (2) in Definition 4.1 (at least not if the Poisson bracket is nontrivial). In fact, the existence problem is highly nontrivial. In the symplectic case, different existence proofs, from different perspectives, were given by Marc De Wilde and Lecomte [52], Omori et al. [53, 54], and Fedosov [55, 56]. The general Poisson case was settled by Kontsevich [57].

\subsection{Equivalence and Classification of Star Products}

Definition 4.2. Given a Poisson manifold $(M,\{\cdot, \cdot\})$. Two star products $\star$ and $\star^{\prime}$ associated to the Poisson structure $\{\cdot, \cdot\}$ are called equivalent if and only if there exists a formal series of linear operators

$$
B=\sum_{i=0}^{\infty} B_{i} v^{i}, \quad B_{i}: C^{\infty}(M) \longrightarrow C^{\infty}(M),
$$

with $B_{0}=i d$ such that

$$
B(f) \star^{\prime} B(g)=B(f \star g) .
$$

For local star products in the general Poisson setting, there are complete classification results. Here I will only consider the symplectic case.

To each local star product $\star$, its Fedosov-Deligne class

$$
\mathrm{cl}(\star) \in \frac{1}{\mathrm{i} v}[\omega]+H_{\mathrm{dR}}^{2}(M)[[v]]
$$

can be assigned. Here $H_{\mathrm{dR}}^{2}(M)$ denotes the 2nd deRham cohomology class of closed 2forms modulo exact forms and $H_{\mathrm{dR}}^{2}(M)[[v]]$ the formal power series with such classes as 
coefficients. Such formal power series are called formal deRham classes. In general we will use $[\alpha]$ for the cohomology class of a form $\alpha$.

This assignment gives a $1: 1$ correspondence between the formal deRham classes and the equivalence classes of star products.

For contractible manifolds, we have $H_{\mathrm{dR}}^{2}(M)=0$ and hence there is up to equivalence exactly one local star product. This yields that locally all local star products of a manifold are equivalent to a certain fixed one, which is called the Moyal product. For these and related classification results, see [58-62].

\subsection{Star Products with Separation of Variables}

For our compact Kähler manifolds, we will have many different and even nonequivalent star products. The question is the following: is there a star product which is given in a natural way? The answer will be yes: the Berezin-Toeplitz star product to be introduced below. First we consider star products respecting the complex structure in a certain sense.

Definition 4.3 (Karabegov [63]). A star product is called star product with separation of variables if and only if

$$
f \star h=f \cdot h, \quad h \star g=h \cdot g,
$$

for every locally defined holomorphic function $g$, antiholomorphic function $f$, and arbitrary function $h$.

Recall that a local star product $\star$ for $M$ defines a star product for every open subset $U$ of $M$. We have just to take the bidifferential operators defining $\star$. Hence it makes sense to talk about $\star$-multiplying with local functions.

Proposition 4.4. A local $\star$ product has the separation of variables property if and only if in the bidifferential operators $C_{k}(\cdot, \cdot)$ for $k \geq 1$ in the first argument only derivatives in holomorphic and in the second argument only derivatives in antiholomorphic directions appear.

In Karabegov's original notation the rôles of the holomorphic and antiholomorphic functions are switched. Bordemann and Waldmann [64] called such star products star products of Wick type. Both Karabegov and Bordemann-Waldmann proved that there exist for every Kähler manifold star products of separation of variables type. In Section 4.8, we will give more details on Karabegov's construction. Bordemann and Waldmann modified Fedosov's method $[55,56]$ to obtain such a star product. See also Reshetikhin and Takhtajan [65] for yet another construction. But I like to point out that in all these constructions the result is only a formal star product without any relation to an operator calculus, which will be given by the Berezin-Toeplitz star product introduced in the next section.

Another warning is in order. The property of being a star product of separation of variables type will not be kept by equivalence transformations. 


\subsection{Berezin-Toeplitz Star Product}

Theorem 4.5. There exists a unique (formal) star product $\star_{\mathrm{BT}}$ for $M$

$$
f \star_{\mathrm{BT}} g:=\sum_{j=0}^{\infty} v^{j} C_{j}(f, g), \quad C_{j}(f, g) \in C^{\infty}(M),
$$

in such a way that for $f, g \in C^{\infty}(M)$ and for every $N \in \mathbb{N}$ we have with suitable constants $K_{N}(f, g)$ for all $m$

$$
\left\|T_{f}^{(m)} T_{g}^{(m)}-\sum_{0 \leq j<N}\left(\frac{1}{m}\right)^{j} T_{C_{j}(f, g)}^{(m)}\right\| \leq K_{N}(f, g)\left(\frac{1}{m}\right)^{N} .
$$

The star product is null on constants and self-adjoint.

This theorem has been proven immediately after [1] was finished. It has been announced in $[66,67]$ and the proof was written up in German in [35]. A complete proof published in English can be found in [30].

For simplicity we might write

$$
T_{f}^{(m)} \cdot T_{g}^{(m)} \sim \sum_{j=0}^{\infty}\left(\frac{1}{m}\right)^{j} T_{C_{j}(f, g)}^{(m)} \quad(m \longrightarrow \infty),
$$

but we will always assume the strong and precise statement of (4.9). The same is assumed for other asymptotic formulas appearing further down in this review.

Next we want to identify this star product. Let $K_{M}$ be the canonical line bundle of $M$, that is, the $n$th exterior power of the holomorphic 1-differentials. The canonical class $\delta$ is the first Chern class of this line bundle, that is, $\delta:=c_{1}\left(K_{M}\right)$. If we take in $K_{M}$ the fibre metric coming from the Liouville form $\Omega$, then this defines a unique connection and further a unique curvature $(1,1)$-form $\omega_{\text {can }}$. In our sign conventions, we have $\delta=\left[\omega_{\text {can }}\right]$.

Together with Karabegov the author showed the following theorem.

Theorem 4.6 (see [29]). (a) The Berezin-Toeplitz star product is a local star product which is of separation of variable type.

(b) Its classifying Deligne-Fedosov class is

$$
\mathrm{cl}\left(\star_{\mathrm{BT}}\right)=\frac{1}{\mathrm{i}}\left(\frac{1}{v}[\omega]-\frac{\delta}{2}\right)
$$

for the characteristic class of the star product $\star_{B T}$.

(c) The classifying Karabegov form associated to the Berezin-Toeplitz star product is

$$
-\frac{1}{v} \omega+\omega_{\text {can }} .
$$


The Karabegov form has not yet defined here. We will introduce it below in Section 4.8. Using $K$-theoretic methods, the formula for $\mathrm{cl}\left(\star_{\mathrm{BT}}\right)$ was also given by Hawkins [68].

\subsection{Star Product of Geometric Quantization}

Tuynman's result (3.21) relates the operators of geometric quantization with Kähler polarization and the BT operators. As the latter define a star product, it can be used to give also a star product $\star_{G Q}$ associated to geometric quantization. Details can be found in [30]. This star product will be equivalent to the BT star product, but it is not of the separation of variables type. The equivalence is given by the $\mathbb{C}[[\mathcal{v}]]$-linear map induced by

$$
B(f):=f-v \frac{\Delta}{2} f=\left(i d-v \frac{\Delta}{2}\right) f
$$

We obtain $B(f) \star_{\mathrm{BT}} B(g)=B\left(f \star_{\mathrm{GQ}} g\right)$.

\subsection{Trace for the BT Star Product}

From (3.18) the following complete asymptotic expansion for $m \rightarrow \infty$ can be deduced [30, 69]:

$$
\operatorname{Tr}^{(m)}\left(T_{f}^{(m)}\right) \sim m^{n}\left(\sum_{j=0}^{\infty}\left(\frac{1}{m}\right)^{j} \tau_{j}(f)\right), \quad \text { with } \tau_{j}(f) \in \mathbb{C}
$$

We define the $\mathbb{C}[[\mathcal{v}]]$-linear map

$$
\operatorname{Tr}: C^{\infty}(M)[[v]] \longrightarrow \mathcal{v}^{-n} \mathbb{C}[[v]], \quad \operatorname{Tr} f:=v^{-n} \sum_{j=0}^{\infty} v^{j} \tau_{j}(f),
$$

where the $\tau_{j}(f)$ are given by the asymptotic expansion (4.14) for $f \in C^{\infty}(M)$ and for arbitrary elements by $\mathbb{C}[[\mathcal{v}]]$-linear extension.

Proposition 4.7 (see [30]). The map Tr is a trace, that is, we have

$$
\operatorname{Tr}(f \star g)=\operatorname{Tr}(g \star f) .
$$

\subsection{Karabegov Quantization}

In $[63,70]$ Karabegov not only gave the notion of separation of variables type, but also a proof of existence of such formal star products for any Kähler manifold, whether compact, noncompact, quantizable, or nonquantizable. Moreover, he classified them completely as individual star product not only up to equivalence.

He starts with $\left(M, \omega_{-1}\right)$ a pseudo-Kähler manifold, that is, a complex manifold with a nondegenerate closed $(1,1)$-form not necessarily positive. 
A formal form $\widehat{\omega}=(1 / v) \omega_{-1}+\omega_{0}+v \omega_{1}+\cdots$ is called a formal deformation of the form $(1 / v) \omega_{-1}$ if the forms $\omega_{r}, r \geq 0$, are closed but not necessarily nondegenerate (1,1)-forms on $M$. It was shown in [63] that all deformation quantizations with separation of variables on the pseudo-Kähler manifold $\left(M, \omega_{-1}\right)$ are bijectively parameterized by the formal deformations of the form $(1 / v) \omega_{-1}$.

Assume that we have such a star product $\left(\mathcal{A}:=C^{\infty}(M)[[v]], \star\right)$. Then for $f, g \in \mathcal{A}$ the operators of left and right multiplications $L_{f}, R_{g}$ are given by $L_{f} g=f \star g=R_{g} f$. The associativity of the star-product $\star$ is equivalent to the fact that $L_{f}$ commutes with $R_{g}$ for all $f, g \in \mathcal{A}$. If a star product is differential, then $L_{f}, R_{g}$ are formal differential operators.

Karabegov constructs his star product associated to the deformation $\widehat{\omega}$ in the following way. First he chooses on every contractible coordinate chart $U \subset M$ (with holomorphic coordinates $\left.\left\{z_{k}\right\}\right)$ its formal potential

$$
\widehat{\Phi}=\left(\frac{1}{v}\right) \Phi_{-1}+\Phi_{0}+v \Phi_{1}+\cdots, \quad \widehat{\omega}=i \partial \bar{\partial} \widehat{\Phi} .
$$

Then construction is done in such a way that we have for the left (right) multiplication operators on $U$

$$
L_{\partial \Phi / \partial z_{k}}=\frac{\partial \Phi}{\partial z_{k}}+\frac{\partial}{\partial z_{k}}, \quad R_{\partial \Phi / \partial \bar{z}_{l}}=\frac{\partial \Phi}{\partial \bar{z}_{l}}+\frac{\partial}{\partial \bar{z}_{l}}
$$

The set $\mathcal{L}(U)$ of all left multiplication operators on $U$ is completely described as the set of all formal differential operators commuting with the point-wise multiplication operators by antiholomorphic coordinates $R_{\bar{z}_{l}}=\bar{z}_{l}$ and the operators $R_{\partial \Phi / \partial \bar{z}_{l}}$. From the knowledge of $\mathcal{L}(U)$, the star product on $U$ can be reconstructed. The local star-products agree on the intersections of the charts and define the global star-product $\star$ on $M$.

We have to mention that this original construction of Karabegov will yield a star product of separation of variable type but with the role of holomorphic and antiholomorphic variables switched. This says for any open subset $U \subset M$ and any holomorphic function $a$ and antiholomorphic function $b$ on $U$ that the operators $L_{a}$ and $R_{b}$ are the operators of point-wise multiplication by $a$ and $b$, respectively, that is, $L_{a}=a$ and $R_{b}=b$.

\subsection{Karabegov's Formal Berezin Transform}

Given such a star products $\star$, Karabegov introduced the formal Berezin transform $I$ as the unique formal differential operator on $M$ such that for any open subset $U \subset M$, holomorphic functions $a$, and antiholomorphic functions $b$ on $U$, the relation $I(a \cdot b)=b \star a$ holds (see [71]). He shows that $I=1+v \Delta+\cdots$, where $\Delta$ is the Laplace operator corresponding to the pseudo-Kähler metric on $M$.

Karabegov considered the following associated star products. First the dual starproduct $\widetilde{\star}$ on $M$ is defined for $f, g \in \mathcal{A}$ by the formula

$$
f \widetilde{\star} g=I^{-1}(I g \star I f) .
$$

It is a star-product with separation of variables on the pseudo-Kähler manifold $\left(M,-\omega_{-1}\right)$. Its formal Berezin transform equals $I^{-1}$, and thus the dual to $\widetilde{\star}$ is $\star$. Note that it is not a star 
product of the same pseudo-Kähler manifold. Denote by $\tilde{\omega}=-(1 / v) \omega_{-1}+\tilde{\omega}_{0}+v \tilde{\omega}_{1}+\cdots$ the formal form parameterizing the star-product $\widetilde{\star}$.

Next, the opposite of the dual star-product, $\star^{\prime}=\widetilde{\star}^{\text {op }}$, is given by the formula

$$
f \star^{\prime} g=I^{-1}(I f \star I g)
$$

It defines a deformation quantization with separation of variables on $M$, but with the roles of holomorphic and antiholomorphic variables swapped-with respect to $\star$. It could be described also as a deformation quantization with separation of variables on the pseudoKähler manifold $\left(\bar{M}, \omega_{-1}\right)$, where $\bar{M}$ is the manifold $M$ with the opposite complex structure. But now the pseudo-Kähler form will be the same. Indeed the formal Berezin transform $I$ establishes an equivalence of deformation quantizations $(\mathcal{A}, \star)$ and $\left(\mathcal{A}, \star^{\prime}\right)$.

How is the relation to the Berezin-Toeplitz star product $\star_{\mathrm{BT}}$ of Theorem 4.5? There exists a certain formal deformation $\widehat{\omega}$ of the form $(1 / v) \omega$ which yields a star product $\star$ in the Karabegov sense. The opposite of its dual will be equal to the Berezin-Toeplitz star product, that is,

$$
\star_{\mathrm{BT}}=\widetilde{\star}^{\mathrm{op}}=\star^{\prime} .
$$

The classifying Karabegov form $\tilde{\omega}$ of $\widetilde{\star}$ will be the form (4.12). Note as $\star$ and $\star_{\mathrm{BT}}$ are equivalent via $I$, we have $\mathrm{cl}(\star)=\mathrm{cl}\left(\star_{\mathrm{BT}}\right)$; see the formula (4.11). We will identify $\widehat{\omega}$ in Section 7.5.

\section{The Disc Bundle and Global Operators}

In this section, we identify the bundles $L^{m}$ over the Kähler manifold $M$ as associated line bundles of one unique $S^{1}$-bundle over $M$. The Toeplitz operator will appear as "modes" of a global Toeplitz operator. A detailed analysis of this global operator will yield a proof of Theorem 3.3 part (b) and part (c).

Moreover, we will need this set-up to discuss coherent states, Berezin symbols, and the Berezin transform in the next sections. For a more detailed presentation, see [35].

\subsection{The Disc Bundle}

We will assume that the quantum line bundle $L$ is already very ample, that is, it has enough global holomorphic sections to embed $M$ into projective space. From the bundle (as the connection $\nabla$ will not be needed anymore, I will drop it in the notation) $(L, h)$, we pass to its dual $(U, k):=\left(L^{*}, h^{-1}\right)$ with dual metric $k$. Inside of the total space $U$, we consider the circle bundle

$$
Q:=\{\lambda \in U \mid k(\lambda, \lambda)=1\}
$$


the (open) disc bundle, and (closed) disc bundle, respectively

$$
D:=\{\lambda \in U \mid k(\lambda, \lambda)<1\}, \quad \bar{D}:=\{\lambda \in U \mid k(\lambda, \lambda) \leq 1\}
$$

Let $\tau: U \rightarrow M$ be the projection (maybe restricted to the subbundles).

For the projective space $\mathbb{P}^{N}(\mathbb{C})$ with the hyperplane section bundle $H$ as quantum line bundle, the bundle $U$ is just the tautological bundle. Its fibre over the point $z \in \mathbb{P}^{N}(\mathbb{C})$ consists of the line in $\mathbb{C}^{N+1}$ which is represented by $z$. In particular, for the projective space the total space of $U$ with the zero section removed can be identified with $\mathbb{C}^{N+1} \backslash\{0\}$. The same picture remains true for the via the very ample quantum line bundle in projective space embedded manifold $M$. The quantum line bundle will be the pull-back of $H$ (i.e., its restriction to the embedded manifold) and its dual is the pull-back of the tautological bundle.

In the following we use $E \backslash 0$ to denote the total space of a vector bundle $E$ with the image of the zero section removed. Starting from the real-valued function $\widehat{k}(\lambda):=k(\lambda, \lambda)$ on $U$, we define $\tilde{a}:=(1 / 2 \mathrm{i})(\partial-\bar{\partial}) \log \widehat{k}$ on $U \backslash 0$ (the derivation is taken with respect to the complex structure on $U$ ) and denote by $\alpha$ its restriction to $Q$. With the help of the quantization condition (2.4), we obtain $d \alpha=\tau^{*} \omega$ (with the deRham differential $d=d_{Q}$ ) and that in fact $\mu=(1 / 2 \pi) \tau^{*} \Omega \wedge \alpha$ is a volume form on $Q$. Indeed $\alpha$ is a contact form for the contact manifold $Q$. As far as the integration is concerned we get

$$
\int_{Q}\left(\tau^{*} f\right) \mu=\int_{M} f \Omega, \quad \forall f \in C^{\infty}(M)
$$

Recall that $\Omega$ is the Liouville volume form on $M$.

\subsection{The Generalized Hardy Space}

With respect to $\mu$, we take the $\mathrm{L}^{2}$-completion $\mathrm{L}^{2}(Q, \mu)$ of the space of functions on $Q$. The generalized Hardy space $\mathscr{t}$ is the closure of the space of those functions in $\operatorname{L}^{2}(Q, \mu)$ which can be extended to holomorphic functions on the whole disc bundle $\bar{D}$. The generalized Szegö projector is the projection

$$
\Pi: \mathrm{L}^{2}(Q, \mu) \longrightarrow \mathscr{\ell}
$$

By the natural circle action, the bundle $Q$ is an $S^{1}$-bundle and the tensor powers of $U$ can be viewed as associated line bundles. The space $\mathscr{H}$ is preserved by the $S^{1}$-action. It can be decomposed into eigenspaces $\mathscr{H}=\prod_{m=0}^{\infty} \mathscr{\ell}^{(m)}$, where $c \in S^{1}$ acts on $\mathscr{H}^{(m)}$ as multiplication by $c^{m}$. The Szegö projector is $S^{1}$ invariant and can be decomposed into its components, the Bergman projectors

$$
\widehat{\Pi}^{(m)}: \mathrm{L}^{2}(Q, \mu) \longrightarrow \mathscr{H}^{(m)} .
$$


Sections of $L^{m}=U^{-m}$ can be identified with functions $\psi$ on $Q$ which satisfy the equivariance condition $\psi(c \lambda)=c^{m} \psi(\lambda)$, that is, which are homogeneous of degree $m$. This identification is given via the map

$$
\gamma_{m}: \mathrm{L}^{2}\left(M, L^{m}\right) \longrightarrow \mathrm{L}^{2}(Q, \mu), \quad s \longmapsto \psi_{s}, \quad \text { where } \psi_{s}(\alpha)=\alpha^{\otimes m}(s(\tau(\alpha))),
$$

which turns out to be an isometry onto its image. On $\mathrm{L}^{2}\left(M, L^{m}\right)$, we have the scalar product (3.2). Restricted to the holomorphic sections, we obtain the isometry

$$
\gamma_{m}: \Gamma_{\mathrm{hol}}\left(M, L^{m}\right) \cong \mathscr{H}^{(m)} .
$$

In the case of $\mathbb{P}^{N}(\mathbb{C})$, this correspondence is nothing else as the identification of the global sections of the $m$ th tensor powers of the hyper plane section bundle with the homogenous polynomial functions of degree $m$ on $\mathbb{C}^{N+1}$.

\subsection{The Toeplitz Structure}

There is the notion of Toeplitz structure $(\Pi, \Sigma)$ as developed by Boutet de Monvel and Guillemin in $[28,72]$. I do not want to present the general theory but only the specialization to our situation. Here $\Pi$ is the Szegö projector (5.4) and $\Sigma$ is the submanifold

$$
\Sigma:=\{t \alpha(\lambda) \mid \lambda \in Q, t>0\} \subset T^{*} Q \backslash 0
$$

of the tangent bundle of $Q$ defined with the help of the 1-form $\alpha$. It turns out that $\Sigma$ is a symplectic submanifold, called a symplectic cone.

A (generalized) Toeplitz operator of order $k$ is an operator $A: \mathscr{l} \rightarrow \mathscr{H}$ of the form

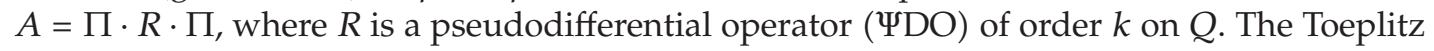
operators constitute a ring. The symbol of $A$ is the restriction of the principal symbol of $R$ (which lives on $T^{*} Q$ ) to $\Sigma$. Note that $R$ is not fixed by $A$, but Guillemin and Boutet de Monvel showed that the symbols are well defined and that they obey the same rules as the symbols of $\Psi D O s$. In particular, the following relations are valid:

$$
\sigma\left(A_{1} A_{2}\right)=\sigma\left(A_{1}\right) \sigma\left(A_{2}\right), \quad \sigma\left(\left[A_{1}, A_{2}\right]\right)=\mathrm{i}\left\{\sigma\left(A_{1}\right), \sigma\left(A_{2}\right)\right\}_{\Sigma} .
$$

Here $\{\cdot, \cdot\}_{\Sigma}$ is the restriction of the canonical Poisson structure of $T^{*} Q$ to $\Sigma$ coming from the canonical symplectic form on $T^{*} Q$.

\subsection{A Sketch of the Proof of Theorem 3.3}

For this we need only to consider the following two generalized Toeplitz operators.

(1) The generator of the circle action gives the operator $D_{\varphi}=(1 / \mathrm{i})(\partial / \partial \varphi)$, where $\varphi$ is the angular variable. It is an operator of order 1 with symbol $t$. It operates on $\mathscr{H}^{(m)}$ as multiplication by $m$. 
(2) For $f \in C^{\infty}(M)$, let $M_{f}$ be the operator on $\mathrm{L}^{2}(Q, \mu)$ corresponding to multiplication with $\tau^{*} f$. We set

$$
T_{f}=\Pi \cdot M_{f} \cdot \Pi: \mathscr{H} \longrightarrow \mathscr{H}
$$

As $M_{f}$ is constant along the fibres of $\tau$, the operator $T_{f}$ commutes with the circle action. Hence we can decompose

$$
T_{f}=\prod_{m=0}^{\infty} T_{f}^{(m)}
$$

where $T_{f}^{(m)}$ denotes the restriction of $T_{f}$ to $\mathscr{L}^{(m)}$. After the identification of $\mathscr{H}^{(m)}$ with $\Gamma_{\text {hol }}\left(M, L^{m}\right)$, we see that these $T_{f}^{(m)}$ are exactly the Toeplitz operators $T_{f}^{(m)}$ introduced in Section 3. We call $T_{f}$ the global Toeplitz operator and the $T_{f}^{(m)}$ the local Toeplitz operators. The operator $T_{f}$ is of order 0 . Let us denote by $\tau_{\Sigma}: \Sigma \subseteq$ $T^{*} Q \rightarrow Q \rightarrow M$ the composition, then we obtain for its symbol $\sigma\left(T_{f}\right)=\tau_{\Sigma}^{*}(f)$.

Now we are able to prove (3.10). First we introduce for a fixed $t>0$

$$
\Sigma_{t}:=\{t \cdot \alpha(\lambda) \mid \lambda \in Q\} \subseteq \Sigma .
$$

It turns out that $\omega_{\Sigma \mid \Sigma_{t}}=-t \tau_{\Sigma}^{*} \omega$. The commutator $\left[T_{f}, T_{g}\right]$ is a Toeplitz operator of order -1 . From the above, we obtain with (5.9) that the symbol of the commutator equals

$$
\sigma\left(\left[T_{f}, T_{g}\right]\right)(t \alpha(\lambda))=\mathrm{i}\left\{\tau_{\Sigma}^{*} f, \tau_{\Sigma}^{*} g\right\}_{\Sigma}(t \alpha(\lambda))=-\mathrm{i} t^{-1}\{f, g\}_{M}(\tau(\lambda))
$$

We consider the Toeplitz operator

$$
A:=D_{\varphi}^{2}\left[T_{f}, T_{g}\right]+\mathrm{i} D_{\varphi} T_{\{f, g\}}
$$

Formally this is an operator of order 1 . Using $\sigma\left(T_{\{f, g\}}\right)=\tau_{\Sigma}^{*}\{f, g\}$ and $\sigma\left(D_{\varphi}\right)=t$, we see that its principal symbol vanishes. Hence it is an operator of order 0 . Now $M$ and hence also $Q$ are compact manifolds. This implies that $A$ is a bounded operator ( $\Psi D O$ s of order 0 on compact manifolds are bounded). It is obviously $S^{1}$-invariant and we can write $A=\prod_{m=0}^{\infty} A^{(m)}$, where $A^{(m)}$ is the restriction of $A$ on the space $\mathscr{L}^{(m)}$. For the norms we get $\left\|A^{(m)}\right\| \leq\|A\|$. But

$$
A^{(m)}=A_{\mid \mathscr{\ell}^{(m)}}=m^{2}\left[T_{f}^{(m)}, T_{g}^{(m)}\right]+\mathrm{i} m T_{\{f, g\}}^{(m)} .
$$

Taking the norm bound and dividing it by $m$, we get part (b) of Theorem 3.3. Using (5.7), the norms involved indeed coincide.

Quite similar, one can prove part (c) of Theorem 3.3 and more general the existence of the coefficients $C_{j}(f, g)$ for the Berezin-Toeplitz star product of Theorem 4.5. See $[30,35]$ for the details. 


\section{Coherent States and Berezin Symbols}

\subsection{Coherent States}

Let the situation be as in the previous section. In particular, $L$ is assumed to be already very ample, $U=L^{*}$ is the dual of the quantum line bundle, $Q \subset U$ the unit circle bundle, and $\tau: Q \rightarrow M$ the projection. In particular, recall the correspondence (5.6) $\psi_{s}(\alpha)=\alpha^{\otimes m}(s \tau(\alpha))$ of $m$-homogeneous functions $\psi_{s}$ on $U$ with sections of $L^{m}$. To obtain this correspondence, we fixed the section $s$ and varied $a$.

Now we do the opposite. We fix $\alpha \in U \backslash 0$ and vary the section $s$. Obviously, this yields a linear form on $\Gamma_{\text {hol }}\left(M, L^{m}\right)$ and hence with the help of the scalar product (3.2), we make the following.

Definition 6.1. (a) The coherent vector (of level $m$ ) associated to the point $\alpha \in U \backslash 0$ is the unique element $e_{\alpha}^{(m)}$ of $\Gamma_{\text {hol }}\left(M, L^{m}\right)$ such that

$$
\left\langle e_{\alpha}^{(m)}, s\right\rangle=\psi_{s}(\alpha)=\alpha^{\otimes m}(s(\tau(\alpha)))
$$

for all $s \in \Gamma_{\text {hol }}\left(M, L^{m}\right)$.

(b) The coherent state (of level $m$ ) associated to $x \in M$ is the projective class

$$
\mathrm{e}_{x}^{(m)}:=\left[e_{\alpha}^{(m)}\right] \in \mathbb{P}\left(\Gamma_{\mathrm{hol}}\left(M, L^{m}\right)\right), \quad \alpha \in \tau^{-1}(x), \quad \alpha \neq 0 .
$$

Of course, we have to show that the object in $(b)$ is well defined. Recall that $\langle\cdot, \cdot\rangle$ denotes the scalar product on the space of global sections $\Gamma_{\infty}\left(M, L^{m}\right)$. In the convention of this review, it will be antilinear in the first argument and linear in the second argument. The coherent vectors are antiholomorphic in $\alpha$ and fulfil

$$
e_{c \alpha}^{(m)}=\bar{c}^{m} \cdot e_{\alpha}^{(m)}, \quad c \in \mathbb{C}^{*}:=\mathbb{C} \backslash\{0\} .
$$

Note that $e_{\alpha}^{(m)} \equiv 0$ would imply that all sections will vanish at the point $x=\tau(\alpha)$. Hence, the sections of $L$ cannot be used to embed $M$ into a projective space, which is a contradiction to the very ampleness of $L$. Hence, $e_{\alpha}^{(m)} \not \equiv 0$ and due to (6.3) the class

$$
\left[e_{\alpha}^{(m)}\right]:=\left\{s \in \Gamma_{\mathrm{hol}}\left(M, L^{m}\right) \mid \exists c \in \mathbb{C}^{*}: s=c \cdot e_{\alpha}^{(m)}\right\}
$$

is a well-defined element of the projective space $\mathbb{P}\left(\Gamma_{\text {hol }}\left(M, L^{m}\right)\right)$, only depending on $x=$ $\tau(\alpha) \in M$.

This kind of coherent states goes back to Berezin. A coordinate independent version and extensions to line bundles were given by Rawnsley [73]. It plays an important role in the work of Cahen et al. on the quantization of Kähler manifolds [74-77], via Berezin's covariant symbols. I will return to this in Section 6.5. In these works, the coherent vectors are parameterized by the elements of $L \backslash 0$. The definition here uses the points of the total space of the dual bundle $U$. It has the advantage that one can consider all tensor powers of $L$ together on an equal footing. 
Definition 6.2. The coherent state embedding is the antiholomorphic embedding

$$
M \longrightarrow \mathbb{P}\left(\Gamma_{\mathrm{hol}}\left(M, L^{m}\right)\right) \cong \mathbb{P}^{N}(\mathbb{C}), \quad x \longmapsto\left[e_{\tau^{-1}(x)}^{(m)}\right]
$$

Here $N=\operatorname{dim} \Gamma_{\text {hol }}\left(M, L^{m}\right)-1$. In this review, in abuse of notation, $\tau^{-1}(x)$ will always denote a non-zero element of the fiber over $x$. The coherent state embedding is up to conjugation the embedding of Section 2.9 with respect to an orthonormal basis of the sections. In [78] further results on the geometry of the coherent state embedding are given.

\subsection{Covariant Berezin Symbols}

We start with the following definition.

Definition 6.3. The covariant Berezin symbol $\sigma^{(m)}(A)$ (of level $m$ ) of an operator $A \in$ $\operatorname{End}\left(\Gamma_{\mathrm{hol}}\left(M, L^{(m)}\right)\right)$ is defined as

$$
\sigma^{(m)}(A): M \longrightarrow \mathbb{C}, \quad x \longmapsto \sigma^{(m)}(A)(x):=\frac{\left\langle e_{\alpha}^{(m)}, A e_{\alpha}^{(m)}\right\rangle}{\left\langle e_{\alpha}^{(m)}, e_{\alpha}^{(m)}\right\rangle}, \quad \alpha \in \tau^{-1}(x)
$$

As the factors appearing in (6.3) will cancel, it is a well-defined function on $M$. If the level $m$ is clear from the context, I will sometimes drop it in the notation.

We consider also the coherent projectors used by Rawnsley

$$
P_{x}^{(m)}=\frac{\left|e_{\alpha}^{(m)}\right\rangle\left\langle e_{\alpha}^{(m)}\right|}{\left\langle e_{\alpha}^{(m)}, e_{\alpha}^{(m)}\right\rangle}, \quad \alpha \in \tau^{-1}(x)
$$

Here we used the convenient bra-ket notation of the physicists. Recall, if $s$ is a section, then

$$
P_{x}^{(m)} s=\frac{\left|e_{\alpha}^{(m)}\right\rangle\left\langle e_{\alpha}^{(m)}, s\right\rangle}{\left\langle e_{\alpha}^{(m)}, e_{\alpha}^{(m)}\right\rangle}=\frac{\left\langle e_{\alpha}^{(m)}, s\right\rangle}{\left\langle e_{\alpha}^{(m)}, e_{\alpha}^{(m)}\right\rangle} e_{\alpha}^{(m)}
$$

Again the projector is well defined on $M$. With its help, the covariant symbol can be expressed as

$$
\sigma^{(m)}(A)=\operatorname{Tr}\left(A P_{x}^{(m)}\right)
$$

From the definition of the symbol, it follows that $\sigma^{(m)}(A)$ is real analytic and

$$
\sigma^{(m)}\left(A^{*}\right)=\overline{\sigma^{(m)}(A)}
$$




\subsection{Rawnsley's $\in$ Function}

Rawnsley [73] introduced a very helpful function on the manifold $M$ relating the local metric in the bundle with the scalar product on coherent states. In our dual description, we define it in the following way.

Definition 6.4. Rawnsley's epsilon function is the function

$$
M \longrightarrow C^{\infty}(M), \quad x \longmapsto \epsilon^{(m)}(x):=\frac{h^{(m)}\left(e_{\alpha}^{(m)}, e_{\alpha}^{(m)}\right)(x)}{\left\langle e_{\alpha}^{(m)}, e_{\alpha}^{(m)}\right\rangle}, \quad \alpha \in \tau^{-1}(x) .
$$

With (6.3), it is clear that it is a well-defined function on M. Furthermore, using (6.1)

$$
0 \neq\left\langle e_{\alpha}^{(m)}, e_{\alpha}^{(m)}\right\rangle=\alpha^{\otimes m}\left(e_{\alpha}^{(m)}(\tau(\alpha))\right)
$$

it follows that

$$
e_{\alpha}^{(m)}(x) \neq 0, \quad \text { for } x=\tau(\alpha) \text {, and } \epsilon^{(m)}>0 .
$$

Hence, we can define the modified measure

$$
\Omega_{\epsilon}^{(m)}(x):=\epsilon^{(m)}(x) \Omega(x)
$$

for the space of functions on $M$ and obtain a modified scalar product $\langle\cdot, \cdot\rangle_{e}^{(m)}$ for $C^{\infty}(M)$.

Proposition 6.5. For $s_{1}, s_{2} \in \Gamma_{\text {hol }}\left(M, L^{m}\right)$, we have

$$
\begin{aligned}
h^{(m)}\left(s_{1}, s_{2}\right)(x) & =\frac{\overline{\left\langle e_{\alpha}^{(m)}, s_{1}\right\rangle}\left\langle e_{\alpha}^{(m)}, s_{2}\right\rangle}{\left\langle e_{\alpha}^{(m)}, e_{\alpha}^{(m)}\right\rangle} \cdot \epsilon^{(m)}(x) \\
& =\left\langle s_{1}, P_{x}^{(m)} s_{2}\right\rangle \cdot \epsilon^{(m)}(x) .
\end{aligned}
$$

Proof. Due to (6.13), we can represent every section $s$ locally at $x$ as $s(x)=\widehat{s}(x) e_{\alpha}^{(m)}$ with a local function $\widehat{s}$. Now

$$
\left\langle e_{\alpha}^{(m)}, s\right\rangle=\alpha^{(m)}\left(\widehat{s}(x) e_{\alpha}^{(m)}(x)\right)=\widehat{s}(x) \alpha^{(m)}\left(e_{\alpha}^{(m)}(x)\right)=\widehat{s}(x)\left\langle e_{\alpha}^{(m)}, e_{\alpha}^{(m)}\right\rangle .
$$

We rewrite $h^{(m)}\left(s_{1}, s_{2}\right)(x)=\overline{\widehat{s}_{1}} s_{2} h^{(m)}\left(e_{\alpha}^{(m)}, e_{\alpha}^{(m)}\right)(x)$, and obtain

$$
h^{(m)}\left(s_{1}, s_{2}\right)(x)=\frac{\overline{\left\langle e_{\alpha}^{(m)}, s_{1}\right\rangle}}{\left\langle e_{\alpha}^{(m)}, e_{\alpha}^{(m)}\right\rangle} \frac{\left\langle e_{\alpha}^{(m)}, s_{2}\right\rangle}{\left\langle e_{\alpha}^{(m)}, e_{\alpha}^{(m)}\right\rangle} \cdot h^{(m)}\left(e_{\alpha}^{(m)}, e_{\alpha}^{(m)}\right)(x) .
$$


From the definition (6.11), the first relation follows. Obviously, it can be rewritten with the coherent projector to obtain the second relation.

There exists another useful description of the epsilon function.

Proposition 6.6. Let $s_{1}, s_{2}, \ldots, s_{k}$ be an arbitrary orthonormal basis of $\Gamma_{\text {hol }}\left(M, L^{m}\right)$. Then

$$
\epsilon^{(m)}(x)=\sum_{j=1}^{k} h^{(m)}\left(s_{j}, s_{j}\right)(x)
$$

Proof. For every vector $\psi$ in a finite-dimensional hermitian vector space with orthonormal basis $s_{j}, j=1, \ldots, k$, the coefficient with respect to the basis element $s_{j}$ is given by $\psi_{j}=$ $\left\langle s_{j}, \psi\right\rangle$. Furthermore, $\langle\psi, \psi\rangle=\|\psi\|^{2}=\sum_{j} \overline{\psi_{j}} \psi_{j}$. Using the relation (6.15) we can rewrite

$$
\sum_{j=1}^{k} h\left(s_{j}, s_{j}\right)(x)=\frac{\epsilon^{(m)}(x)}{\left\langle e_{\alpha}^{(m)}, e_{\alpha}^{(m)}\right\rangle} \sum_{j=1}^{k} \overline{\left\langle e_{\alpha}^{(m)}, s_{j}\right\rangle}\left\langle e_{\alpha}^{(m)}, s_{j}\right\rangle .
$$

Hence the claim follows.

In certain special cases, the functions $\epsilon^{(m)}$ will be constant as a function of the points of the manifold. In this case, we can apply Proposition 6.11 below for $A=i d$, the identity operator, and obtain

$$
\epsilon^{(m)}=\frac{\operatorname{dim} \Gamma_{\mathrm{hol}}\left(M, L^{m}\right)}{\operatorname{vol} M} .
$$

Here vol $M$ denotes the volume of the manifold with respect to the Liouville measure. Now the question arises when $\epsilon^{(m)}$ will be constant, respectively, when the measure $\Omega_{e}^{(m)}$ will be the standard measure (up to a scalar). From Proposition 6.6, it follows that if there is a transitive group action on the manifold and everything, for example, Kähler form, bundle, metric, is homogenous with respect to the action this will be the case. An example is given by $M=$ $\mathbb{P}^{N}(\mathbb{C})$. By a result of Rawnsley [73], respectively, Cahen et al. [74], $\epsilon^{(m)} \equiv$ const if and only if the quantization is projectively induced. This means that under the conjugate of the coherent state embedding, the Kähler form $\omega$ of $M$ coincides with the pull-back of the Fubini-Study form. Note that in general this is not the case; see Section 7.6.

\subsection{Contravariant Berezin Symbols}

Recall the modified Liouville measure (6.14) and modified scalar product for the functions on $M$ introduced in the last subsection. 
Definition 6.7. Given an operator $A \in \operatorname{End}\left(\Gamma_{\text {hol }}\left(M, L^{(m)}\right)\right)$, then a contravariant Berezin symbol $\breve{\sigma}^{(m)}(A) \in C^{\infty}(M)$ of $A$ is defined by the representation of the operator $A$ as integral

$$
A=\int_{M} \check{\sigma}^{(m)}(A)(x) P_{x}^{(m)} \Omega_{\epsilon}^{(m)}(x)
$$

if such a representation exists.

Proposition 6.8. The Toeplitz operator $T_{f}^{(m)}$ admits a representation (6.21) with

$$
\check{\sigma}^{(m)}\left(T_{f}^{(m)}\right)=f
$$

that is, the function $f$ is a contravariant symbol of the Toeplitz operator $T_{f}^{(m)}$. Moreover, every operator $A \in \operatorname{End}\left(\Gamma_{\mathrm{hol}}\left(M, L^{(m)}\right)\right)$, has a contravariant symbol.

Proof. Let $f \in C^{\infty}(M)$ and set

$$
A:=\int_{M} f(x) P_{x}^{(m)} \Omega_{e}^{(m)}(x)
$$

then $\check{\sigma}^{(m)}(A)=f$. For arbitrary $s_{1}, s_{2} \in \Gamma_{\text {hol }}\left(M, L^{m}\right)$, we calculate (using (6.15))

$$
\begin{aligned}
\left\langle s_{1}, A s_{2}\right\rangle & =\int_{M} f(x)\left\langle s_{1}, P_{x}^{(m)} s_{2}\right\rangle \Omega_{e}^{(m)}(x) \\
& =\int_{M} f(x) h^{(m)}\left(s_{1}, s_{2}\right)(x) \Omega(x) \\
& =\int_{M} h^{(m)}\left(s_{1}, f s_{2}\right)(x) \Omega(x) \\
& =\left\langle s_{1}, f s_{2}\right\rangle=\left\langle s_{1}, T_{f}^{(m)} s_{2}\right\rangle .
\end{aligned}
$$

Hence $T_{f}^{(m)}=A$. As the Toeplitz map is surjective (Proposition 3.6), every operator is a Toeplitz operator, hence has a contravariant symbol. defined.

Note that given an operator its contravariant symbol on a fixed level $m$ is not uniquely We introduce on $\operatorname{End}\left(\Gamma_{\text {hol }}\left(M, L^{(m)}\right)\right)$ the Hilbert-Schmidt norm

$$
\langle A, C\rangle_{\mathrm{HS}}=\operatorname{Tr}\left(A^{*} \cdot C\right)
$$

Theorem 6.9. The Toeplitz map $f \rightarrow T_{f}^{(m)}$ and the covariant symbol map $A \rightarrow \sigma^{(m)}(A)$ are adjoint:

$$
\left\langle A, T_{f}^{(m)}\right\rangle_{\mathrm{HS}}=\left\langle\sigma^{(m)}(A), f\right\rangle_{\epsilon}^{(m)}
$$


Proof.

$$
\left\langle A, T_{f}^{(m)}\right\rangle=\operatorname{Tr}\left(A^{*} \cdot T_{f}^{(m)}\right)=\operatorname{Tr}\left(A^{*} \int_{M} f(x) P_{x}^{(m)} \Omega_{\epsilon}^{(m)}(x)\right)=\int_{M} f(x) \operatorname{Tr}\left(A^{*} \cdot P_{x}^{(m)}\right) \Omega_{\epsilon}^{(m)}(x) .
$$

Now applying Definition 6.7 and (6.10)

$$
\left\langle A, T_{f}^{(m)}\right\rangle=\int_{M} f(x) \sigma^{(m)}\left(A^{*}\right) \Omega_{e}^{(m)}(x)=\int_{M} \overline{\sigma^{(m)}(A)}(x) f(x) \Omega_{e}^{(m)}(x)=\left\langle\sigma^{(m)}(A), f(x)\right\rangle_{\epsilon}^{(m)} .
$$

As every operator has a contravariant symbol, we can also conclude

$$
\langle A, B\rangle_{\mathrm{HS}}=\left\langle\sigma^{(m)}(A), \check{\sigma}^{(m)}(B)\right\rangle_{\epsilon}^{(m)}
$$

From Theorem 6.9 by using the surjectivity of the Toeplitz map, we get the following proposition.

Proposition 6.10. The covariant symbol map $\sigma^{(m)}$ is injective.

Another application is the following.

\section{Proposition 6.11.}

$$
\operatorname{Tr} A=\int_{M} \sigma^{(m)}(A) \Omega_{e}^{(m)}
$$

Proof. We use Id $=T_{1}$ and by (6.26) $\operatorname{Tr} A=\langle A, \mathrm{Id}\rangle_{\mathrm{HS}}=\left\langle\sigma^{(m)}(A), 1\right\rangle_{e}^{(m)}$.

\subsection{Berezin Star Product}

Under certain very restrictive conditions, Berezin covariant symbols can be used to construct a star product, called the Berezin star product. Recall that Proposition 6.10 says that the linear symbol map

$$
\sigma^{(m)}: \operatorname{End}\left(\Gamma_{\text {hol }}\left(M, L^{(m)}\right)\right) \rightarrow C^{\infty}(M)
$$

is injective. Its image is a subspace $\mathscr{A}^{(m)}$ of $C^{\infty}(M)$, called the subspace of covariant symbols of level $m$. If $\sigma^{(m)}(A)$ and $\sigma^{(m)}(B)$ are elements of this subspace the operators, $A$ and $B$ will 
be uniquely fixed. Hence also $\sigma^{(m)}(A \cdot B)$. Now one takes

$$
\sigma^{(m)}(A) \star_{(m)} \sigma^{(m)}(B):=\sigma^{(m)}(A \cdot B)
$$

as a definition for an associative and noncommutative product $\star_{(m)}$ on $\mathcal{A}^{(m)}$.

It is even possible to give an analytic expression for the resulting symbol. For this we introduce the two-point function

$$
\psi^{(m)}(x, y)=\frac{\left\langle e_{\alpha}^{(m)}, e_{\beta}^{(m)}\right\rangle\left\langle e_{\beta}^{(m)}, e_{\alpha}^{(m)}\right\rangle}{\left\langle e_{\alpha}^{(m)}, e_{\alpha}^{(m)}\right\rangle\left\langle e_{\beta}^{(m)}, e_{\beta}^{(m)}\right\rangle}
$$

with $\alpha=\tau^{-1}(x)=x$ and $\beta=\tau^{-1}(y)$. This function is well defined on $M \times M$. Furthermore, we have the two-point symbol

$$
\sigma^{(m)}(A)(x, y)=\frac{\left\langle e_{\alpha}^{(m)}, A e_{\beta}^{(m)}\right\rangle}{\left\langle e_{\alpha}^{(m)}, e_{\beta}^{(m)}\right\rangle} .
$$

It is the analytic extension of the real-analytic covariant symbol. It is well defined on an open dense subset of $M \times M$ containing the diagonal. Using (6.15), we express

$$
\begin{aligned}
\sigma^{(m)}(A \cdot B)(x) & =\frac{\left\langle e_{\alpha}^{(m)}, A \cdot B e_{\alpha}^{(m)}\right\rangle}{\left\langle e_{\alpha}^{(m)}, e_{\alpha}^{(m)}\right\rangle} \\
& =\frac{\left\langle A^{*} e_{\alpha}^{(m)}, B e_{\alpha}^{(m)}\right\rangle}{\left\langle e_{\alpha}^{(m)}, e_{\alpha}^{(m)}\right\rangle} \\
& =\int_{M} h^{(m)}\left(A^{*} e_{\alpha}^{(m)}, B e_{\alpha}^{(m)}\right)(y) \frac{\Omega(y)}{\left\langle e_{\alpha}^{(m)}, e_{\alpha}^{(m)}\right\rangle} \\
& =\int_{M} \frac{\left\langle e_{\alpha}^{(m)}, A e_{\beta}^{(m)}\right\rangle\left\langle e_{\beta}^{(m)}, B e_{\alpha}^{(m)}\right\rangle}{\left\langle e_{\beta}^{(m)}, e_{\beta}^{(m)}\right\rangle} \frac{\epsilon^{(m)}(y) \Omega(y)}{\left\langle e_{\alpha}^{(m)}, e_{\alpha}^{(m)}\right\rangle} \\
& =\int_{M} \sigma^{(m)}(A)(x, y) \sigma^{(m)}(B)(y, x) \cdot \psi^{(m)}(x, y) \cdot \epsilon^{(m)}(y) \Omega(y) .
\end{aligned}
$$

The crucial problem is how to relate different levels $m$ to define for all possible symbols a unique product not depending on $m$. In certain special situations like these studied by Berezin himself [36] and Cahen et al. [74], the subspaces are nested into each other and the union $\mathcal{A}=\bigcup_{m \in \mathbb{N}} \mathscr{A}^{(m)}$ is a dense subalgebra of $C^{\infty}(M)$. Indeed, in the cases considered, the manifold is a homogenous manifold and the epsilon function $\epsilon^{(m)}$ is a constant. A detailed analysis shows that in this case a star product is given. 
For further examples, for which this method works (not necessarily compact), see other articles by Cahen et al. [75-77]. For related results, see also work of Moreno and OrtegaNavarro [79, 80]. In particular, also the work of Engliš [46, 81-83]. Reshetikhin and Takhtajan [65] gave a construction of a (formal) star product using formal integrals in the spirit of the Berezin's covariant symbol construction.

\section{Berezin Transform}

\subsection{The Definition}

Starting from $f \in C^{\infty}(M)$, we can assign to it its Toeplitz operator $T_{f}^{(m)} \in \operatorname{End}\left(\Gamma_{\text {hol }}\left(M, L^{(m)}\right)\right)$ and then assign to $T_{f}^{(m)}$ the covariant symbol $\sigma^{(m)}\left(T_{f}^{(m)}\right)$. It is again an element of $C^{\infty}(M)$.

Definition 7.1. The map

$$
C^{\infty}(M) \longrightarrow C^{\infty}(M), \quad f \longmapsto I^{(m)}(f):=\sigma^{(m)}\left(T_{f}^{(m)}\right)
$$

is called the Berezin transform (of level $m$ ).

From the point of view of Berezin's approach, the operator $T_{f}^{(m)}$ has as a contravariant symbol $f$. Hence $I^{(m)}$ gives a correspondence between contravariant symbols and covariant symbols of operators. The Berezin transform was introduced and studied by Berezin [36] for certain classical symmetric domains in $\mathbb{C}^{n}$. These results were extended by Unterberger and Upmeier [84]; see also Engliš [46, 81, 82] and Engliš and Peetre [85]. Obviously, the Berezin transform makes perfect sense in the compact Kähler case which we consider here.

\subsection{The Asymptotic Expansion}

The results presented here are joint work with Karabegov [29]. See also [86] for an overview.

Theorem 7.2. Given $x \in M$, then the Berezin transform $I^{(m)}(f)$ evaluated at the point $x$ has a complete asymptotic expansion in powers of $1 / m$ as $m \rightarrow \infty$

$$
I^{(m)}(f)(x) \sim \sum_{i=0}^{\infty} I_{i}(f)(x) \frac{1}{m^{i}}
$$

where $I_{i}: C^{\infty}(M) \rightarrow C^{\infty}(M)$ are maps with

$$
I_{0}(f)=f, \quad I_{1}(f)=\Delta f .
$$

Here the $\Delta$ is the usual Laplacian with respect to the metric given by the Kähler form $\omega$. 
Complete asymptotic expansion means the following. Given $f \in C^{\infty}(M), x \in M$, and an $r \in \mathbb{N}$, then there exists a positive constant $A$ such that

$$
\left|I^{(m)}(f)(x)-\sum_{i=0}^{r-1} I_{i}(f)(x) \frac{1}{m^{i}}\right|_{\infty} \leq \frac{A}{m^{r}} .
$$

In Section 7.4, I will give some remarks on the proof but before I present you a nice application.

\subsection{Norm Preservation of the BT Operators}

In [87] I conjectured (7.2) (which is now a mathematical result) and showed how such an asymptotic expansion supplies a different proof of Theorem 3.3, part (a). For completeness, I reproduce the proof here.

\section{Proposition 7.3.}

$$
\left|I^{(m)}(f)\right|_{\infty}=\left|\sigma^{(m)}\left(T_{f}^{(m)}\right)\right|_{\infty} \leq\left\|T_{f}^{(m)}\right\| \leq|f|_{\infty}
$$

Proof. Using Cauchy-Schwarz inequality, we calculate $(x=\tau(\alpha))$

$$
\left|\sigma^{(m)}\left(T_{f}^{(m)}\right)(x)\right|^{2}=\frac{\left|\left\langle e_{\alpha}^{(m)}, T_{f}^{(m)} e_{\alpha}^{(m)}\right\rangle\right|^{2}}{\left\langle e_{\alpha}^{(m)}, e_{\alpha}^{(m)}\right\rangle^{2}} \leq \frac{\left\langle T_{f}^{(m)} e_{\alpha}^{(m)}, T_{f}^{(m)} e_{\alpha}^{(m)}\right\rangle}{\left\langle e_{\alpha}^{(m)}, e_{\alpha}^{(m)}\right\rangle} \leq\left\|T_{f}^{(m)}\right\|^{2} .
$$

Here the last inequality follows from the definition of the operator norm. This shows the first inequality in (7.5). For the second inequality, introduce the multiplication operator $M_{f}^{(m)}$ on $\Gamma_{\infty}\left(M, L^{m}\right)$. Then $\left\|T_{f}^{(m)}\right\|=\left\|\Pi^{(m)} M_{f}^{(m)} \Pi^{(m)}\right\| \leq\left\|M_{f}^{(m)}\right\|$ and for $\varphi \in \Gamma_{\infty}\left(M, L^{m}\right), \varphi \neq 0$

$$
\frac{\left\|M_{f}^{(m)} \varphi\right\|^{2}}{\|\varphi\|^{2}}=\frac{\int_{M} h^{(m)}(f \varphi, f \varphi) \Omega}{\int_{M} h^{(m)}(\varphi, \varphi) \Omega}=\frac{\int_{M} f(z) \overline{f(z)} h^{(m)}(\varphi, \varphi) \Omega}{\int_{M} h^{(m)}(\varphi, \varphi) \Omega} \leq|f|_{\infty}^{2} .
$$

Hence,

$$
\left\|T_{f}^{(m)}\right\| \leq\left\|M_{f}^{(m)}\right\|=\sup _{\varphi \neq 0} \frac{\left\|M_{f}^{(m)} \varphi\right\|}{\|\varphi\|} \leq|f|_{\infty}
$$

Proof (Theorem 3.3 part (a)). Choose as $x_{e} \in M$ a point with $\left|f\left(x_{e}\right)\right|=|f|_{\infty}$. From the fact that the Berezin transform has as a leading term the identity, it follows that $\left|\left(I^{(m)} f\right)\left(x_{e}\right)-f\left(x_{e}\right)\right| \leq$ $C / m$ with a suitable constant $C$. Hence, $\left\|f\left(x_{e}\right)|-|\left(I^{(m)} f\right)\left(x_{e}\right)\right\| \leq C / m$ and

$$
|f|_{\infty}-\frac{C}{m}=\left|f\left(x_{e}\right)\right|-\frac{C}{m} \leq\left|\left(I^{(m)} f\right)\left(x_{e}\right)\right| \leq\left|I^{(m)} f\right|_{\infty}
$$


Putting (7.5) and (7.9) together, we obtain

$$
|f|_{\infty}-\frac{C}{m} \leq\left\|T_{f}^{(m)}\right\| \leq|f|_{\infty}
$$

\subsection{Bergman Kernel}

To understand the Berezin transform better, we have to study the Bergman kernel. Recall from Section 5, the Szegö projectors $\Pi: \mathrm{L}^{2}(Q, \mu) \rightarrow \mathscr{H}$ and its components $\widehat{\Pi}^{(m)}: \mathrm{L}^{2}(Q, \mu) \rightarrow$ $\mathscr{H}^{(m)}$, the Bergman projectors. The Bergman projectors have smooth integral kernels, the Bergman kernels $\boldsymbol{B}_{m}(\alpha, \beta)$ defined on $Q \times Q$, that is,

$$
\widehat{\Pi}^{(m)}(\psi)(\alpha)=\int_{Q} \boldsymbol{B}_{m}(\alpha, \beta) \psi(\beta) \mu(\beta) .
$$

The Bergman kernels can be expressed with the help of the coherent vectors.

\section{Proposition 7.4.}

$$
B_{m}(\alpha, \beta)=\psi_{e_{\beta}^{(m)}}(\alpha)=\overline{\psi_{e_{\alpha}^{(m)}}^{(\beta)}}=\left\langle e_{\alpha}^{(m)}, e_{\beta}^{(m)}\right\rangle
$$

For the proofs of this and the following propositions, see [29] or [86].

Let $x, y \in M$ and choose $\alpha, \beta \in Q$ with $\tau(\alpha)=x$ and $\tau(\beta)=y$, then the functions

$$
\begin{gathered}
u_{m}(x):=\mathcal{B}_{m}(\alpha, \alpha)=\left\langle e_{\alpha}^{(m)}, e_{\alpha}^{(m)}\right\rangle, \\
v_{m}(x, y):=\mathcal{B}_{m}(\alpha, \beta) \cdot \mathcal{B}_{m}(\beta, \alpha)=\left\langle e_{\alpha}^{(m)}, e_{\beta}^{(m)}\right\rangle \cdot\left\langle e_{\beta}^{(m)}, e_{\alpha}^{(m)}\right\rangle
\end{gathered}
$$

are well defined on $M$ and on $M \times M$, respectively. The following proposition gives an integral representation of the Berezin transform.

\section{Proposition 7.5.}

$$
\begin{aligned}
\left(I^{(m)}(f)\right)(x) & =\frac{1}{\boldsymbol{B}_{m}(\alpha, \alpha)} \int_{Q} \boldsymbol{B}_{m}(\alpha, \beta) \boldsymbol{B}_{m}(\beta, \alpha) \tau^{*} f(\beta) \mu(\beta) \\
& =\frac{1}{u_{m}(x)} \int_{M} v_{m}(x, y) f(y) \Omega(y) .
\end{aligned}
$$

Typically, asymptotic expansions can be obtained using stationary phase integrals. But for such an asymptotic expansion of the integral representation of the Berezin transform, we will not only need an asymptotic expansion of the Bergman kernel along the diagonal (which is well known) but in a neighbourhood of it. This is one of the key results obtained in [29]. It is based on works of Boutet de Monvel and Sjöstrand [88] on the Szegö kernel and in generalization of a result of Zelditch [89] on the Bergman kernel on the diagonal. The integral 
representation is used then to prove the existence of the asymptotic expansion of the Berezin transform.

Having such an asymptotic expansion, it still remains to identify its terms. As it was explaining in Section 4.8, Karabegov assigns to every formal deformation quantizations with the "separation of variables" property a formal Berezin transform I. In [29] it is shown that there is an explicitly specified star product $\star$ (see [29, Theorem 5.9]) with associated formal Berezin transform such that if we replace $1 / m$ by the formal variable $v$ in the asymptotic expansion of the Berezin transform $I^{(m)} f(x)$ we obtain $I(f)(x)$. This finally proves Theorem 7.2. We will exhibit the star product $\star$ in the next section.

\subsection{Identification of the BT Star Product}

Moreover in [29] there is another object introduced, the twisted product

$$
R^{(m)}(f, g):=\sigma^{(m)}\left(T_{f}^{(m)} \cdot T_{g}^{(m)}\right) .
$$

Also for it the existence of a complete asymptotic expansion was shown. It was identified with a twisted formal product. This allows the identification of the BT star product with a special star product within the classification of Karabegov. From this identification, the properties of Theorem 4.6 of locality, separation of variables type, and the calculation to the classifying forms and classes for the BT star product follow.

As already announced in Section 4.8, the BT star product $\star_{\mathrm{BT}}$ is the opposite of the dual star product of a certain star product $\star$. To identify $\star$ we will give its classifying Karabegov form $\widehat{\omega}$. As already mentioned above, Zelditch [89] proved that the the function $u_{m}$ (7.13) has a complete asymptotic expansion in powers of $1 / \mathrm{m}$. In detail he showed

$$
u_{m}(x) \sim m^{n} \sum_{k=0}^{\infty} \frac{1}{m^{k}} b_{k}(x), \quad b_{0}=1 .
$$

If we replace in the expansion $1 / \mathrm{m}$ by the formal variable $v$, we obtain a formal function $s$ defined by

$$
e^{s}(x)=\sum_{k=0}^{\infty} v^{k} b_{k}(x)
$$

Now take as formal potential (4.17)

$$
\widehat{\Phi}=\frac{1}{v} \Phi_{-1}+s,
$$


where $\Phi_{-1}$ is the local Kähler potential of the Kähler form $\omega=\omega_{-1}$. Then $\widehat{\omega}=i \partial \bar{\partial} \widehat{\Phi}$. It might be also written in the form

$$
\widehat{\omega}=\frac{1}{v} \omega+\mathbb{F}\left(i \partial \bar{\partial} \log 乃_{m}(\alpha, \alpha)\right)
$$

Here we denote the replacement of $1 / m$ by the formal variable $v$ by the symbol $\mathbb{F}$.

\subsection{Pullback of the Fubini-Study Form}

Starting from the Kähler manifold $(M, \omega)$ and after choosing an orthonormal basis of the space $\Gamma_{\text {hol }}\left(M, L^{m}\right)$, we obtain an embedding

$$
\phi^{(m)}: M \longrightarrow \mathbb{P}^{N(m)}
$$

of $M$ into projective space of dimension $N(m)$. On $\mathbb{P}^{N(m)}$ we have the standard Kähler form, the Fubini-Study form $\omega_{\mathrm{FS}}$. The pull-back $\left(\phi^{(m)}\right)^{*} \omega_{\mathrm{FS}}$ will not depend on the orthogonal basis chosen for the embedding. But in general it will not coincide with a scalar multiple of the Kähler form $\omega$ we started with (see [78] for a thorough discussion of the situation).

It was shown by Zelditch [89], by generalizing a result of Tian [90], that $\left(\Phi^{(m)}\right)^{*} \omega_{\mathrm{FS}}$ admits a complete asymptotic expansion in powers of $1 / m$ as $m \rightarrow \infty$. In fact it is related to the asymptotic expansion of the Bergman kernel (7.13) along the diagonal. The pull-back can be given as [89, Proposition 9]

$$
\left(\phi^{(m)}\right)^{*} \omega_{\mathrm{FS}}=m \omega+\mathrm{i} \partial \bar{\partial} \log u_{m}(x)
$$

If we again replace $1 / m$ by $v$, we obtain via (7.20) the Karabegov form introduced in Section 4.8

$$
\widehat{\omega}=\mathbb{F}\left(\left(\phi^{(m)}\right)^{*} \omega_{\mathrm{FS}}\right)
$$

\section{References}

[1] M. Bordemann, E. Meinrenken, and M. Schlichenmaier, "Toeplitz quantization of Kähler manifolds and $\operatorname{gl}(n), n \rightarrow \infty$ limits," Communications in Mathematical Physics, vol. 165, no. 2, pp. 281-296, 1994.

[2] J. Madore, "The fuzzy sphere," Classical and Quantum Gravity, vol. 9, no. 1, pp. 69-87, 1992.

[3] A. P. Balachandran, B. P. Dolan, J. Lee, X. M., and D. O'Connor, "Fuzzy complex projective spaces and their star-products," Journal of Geometry and Physics, vol. 43, no. 2-3, pp. 184-204, 2002.

[4] A. P. Balachandran, S. Kurkcuoglu, and S. Vaidya, Lectures on Fuzzy and Fuzzy SUSY Physics, World Scientific, Singapore, 2007.

[5] C. I. Lazaroiu, D. McNamee, and Sämann, “Generalized Berezin quantization, Bergman metric and fuzzy laplacians," Journal of High Energy Physics. In press.

[6] B. P. Dolan, I. Huet, S. Murray, and D. O'Connor, "Noncommutative vector bundles over fuzzy $\mathbb{C P}^{N}$ and their covariant derivatives," Journal of High Energy Physics, vol. 7, article 007, 2007.

[7] P. Aschieri, J. Madore, P. Manousselis, and G. Zoupanos, "Dimensional reduction over fuzzy coset spaces," Journal of High Energy Physics, vol. 2004, no. 4, article 034, 2004.

[8] H. Grosse, M. Maceda, J. Madore, and H. Steinacker, "Fuzzy instantons," International Journal of Modern Physics A, vol. 17, no. 15, pp. 2095-2111, 2002. 
[9] H. Grosse and H. Steinacker, "Finite gauge theory on fuzzy $\mathbb{C P}^{2}$," Nuclear Physics B, vol. 704, p. 145, 2005.

[10] U. Carow-Watamura, H. Steinacker, and S. Watamura, "Monopole bundles over fuzzy complex projective spaces," Journal of Geometry and Physics, vol. 54, no. 4, pp. 373-399, 2005.

[11] J. E. Andersen, "Asymptotic faithfulness of the quantum $S U(n)$ representations of the mapping class groups," Annals of Mathematics, vol. 163, no. 1, pp. 347-368, 2006.

[12] J. E. Andersen, "Deformation quantization and geometric quantization of abelian moduli spaces," Communications in Mathematical Physics, vol. 255, no. 3, pp. 727-745, 2005.

[13] M. Schlichenmaier, "Berezin-Toeplitz quantization of the moduli space of flat $S U(N)$ connections," Journal of Geometry and Symmetry in Physics, vol. 9, pp. 33-44, 2007.

[14] J. E. Andersen, “Mapping class groups do not have property (T)," http://arxiv.org/abs/0706.2184.

[15] J. E. Andersen and N. L. Gammelgaard, “Hitchin's projectively flat connection, Toeplitz operators and the asymptotic expansion of TQFT curve operators," http://arxiv.org/abs/0903.4091.

[16] J. E. Andersen and K. Ueno, "Abelian conformal field theory and determinant bundles," International Journal of Mathematics, vol. 18, no. 8, pp. 919-993, 2007.

[17] J. E. Andersen and K. Ueno, "Geometric construction of modular functors from conformal field theory," Journal of Knot Theory and Its Ramifications, vol. 16, no. 2, pp. 127-202, 2007.

[18] X. Ma and G. Marinescu, Holomorphic Morse Inequalities and Bergman Kernels, vol. 254 of Progress in Mathematics, Birkhäuser, Basel, Switzerland, 2007.

[19] X. Ma and G. Marinescu, "Toeplitz operators on symplectic manifolds," Journal of Geometric Analysis, vol. 18, no. 2, pp. 565-611, 2008.

[20] L. Charles, "Toeplitz operators and Hamiltonian torus actions," Journal of Functional Analysis, vol. 236, no. 1, pp. 299-350, 2006.

[21] S. T. Ali and H.-D. Doebner, “Ordering problem in quantum mechanics: prime quantization and a physical interpretation," Physical Review A, vol. 41, no. 3, pp. 1199-1210, 1990.

[22] S. T. Ali and M. Engliš, "Quantization methods: a guide for physicists and analysts," Reviews in Mathematical Physics, vol. 17, no. 4, pp. 391-490, 2005.

[23] S. T. Ali, "Quantization techniques: a quick overview," in Contemporary Problems in Mathematical Physics, pp. 3-78, World Scientific, River Edge, NJ, USA, 2002.

[24] D. Sternheimer, "Deformation quantization: twenty years after," in Particles, Fields, and Gravitation, vol. 453 of AIP Conference Proceedings, pp. 107-145, The American Institute of Physics, Woodbury, NY, USA, 1998.

[25] G. Dito and D. Sternheimer, "Deformation quantization: genesis, developments and metamorphoses," in Deformation Quantization, vol. 1 of IRMA Lectures in Mathematics and Theoretical Physics, pp. 9-54, Walter de Gruyter, Berlin, Germany, 2002.

[26] M. Schlichenmaier, An Introduction to Riemann Surfaces, Algebraic Curves and Moduli Spaces, Theoretical and Mathematical Physics, Springer, Berlin, Germany, 2nd edition, 2007.

[27] R. Abraham and J. E. Marsden, Foundations of Mechanics, Addison Wesley, Redwood City, Calif, USA, 1985.

[28] L. Boutet de Monvel and V. Guillemin, The Spectral Theory of Toeplitz Operators, Princeton University Press, Princeton, NJ, USA, 1981.

[29] A. V. Karabegov and M. Schlichenmaier, "Identification of Berezin-Toeplitz deformation quantization," Journal für die Reine und Angewandte Mathematik, vol. 540, pp. 49-76, 2001.

[30] M. Schlichenmaier, "Deformation quantization of compact Kähler manifolds by Berezin-Toeplitz quantization," in Proceedings of the Conférence Moshé Flato, G. Dito and D. Sternheimer, Eds., vol. 22 of Mathematical Physics Studies, pp. 289-306, Kluwer Academic Publishers, Dordrecht, The Netherlands, 2000.

[31] M. Rieffel, "Questions on quantization," in Operator Algebras and Operator Theory, vol. 228 of Contemporary Mathematics, pp. 315-326, American Mathematical Society, Providence, RI, USA, 1998.

[32] N. P. Landsman, Mathematical Topics between Classical and Quantum Mechanics, Springer Monographs in Mathematics, Springer, New York, NY, USA, 1998.

[33] G. M. Tuynman, "Generalized Bergman kernels and geometric quantization," Journal of Mathematical Physics, vol. 28, no. 3, pp. 573-583, 1987.

[34] M. Bordemann, J. Hoppe, P. Schaller, and M. Schlichenmaier, "gl( $\infty)$ and geometric quantization," Communications in Mathematical Physics, vol. 138, no. 2, pp. 209-244, 1991. 
[35] M. Schlichenmaier, Zwei Anwendungen algebraisch-geometrischer Methoden in der theoretischen Physik: Berezin-Toeplitz-Quantisierung und globale Algebren der zweidimensionalen konformen Feldtheorie, Habiliationsschrift Universität Mannheim, 1996.

[36] F. A. Berezin, "Quantization in complex symmetric spaces," Izvestiya Akademii Nauk SSSR, vol. 9, no. 2, pp. 341-379, 1975.

[37] H. Upmeier, "Toeplitz $C^{*}$-algebras on bounded symmetric domains," Annals of Mathematics, vol. 119, no. 3, pp. 549-576, 1984.

[38] H. Upmeier, "Toeplitz operators on symmetric Siegel domains," Mathematische Annalen, vol. 271, no. 3, pp. 401-414, 1985.

[39] H. Upmeier, "Fredholm indices for Toeplitz operators on bounded symmetric domains," American Journal of Mathematics, vol. 110, no. 5, pp. 811-832, 1988.

[40] H. Upmeier, "Toeplitz C*-algebras and noncommutative duality," Journal of Operator Theory, vol. 26, no. 2, pp. 407-432, 1991.

[41] H. Upmeier, Toeplitz Operators and Index Theory in Several Complex Variables, vol. 81 of Operator Theory: Advances and Applications, Birkhäuser, Basel, Switzerland, 1996.

[42] C. A. Berger and L. A. Coburn, "Toeplitz operators and quantum mechanics," Journal of Functional Analysis, vol. 68, no. 3, pp. 273-299, 1986.

[43] L. A. Coburn, "Deformation estimates for the Berezin-Toeplitz quantization," Communications in Mathematical Physics, vol. 149, no. 2, pp. 415-424, 1992.

[44] S. Klimek and A. Lesniewski, "Quantum Riemann surfaces. I. The unit disc," Communications in Mathematical Physics, vol. 146, no. 1, pp. 103-122, 1992.

[45] S. Klimek and A. Lesniewski, "Quantum Riemann surfaces. II. The discrete series," Communications in Mathematical Physics, vol. 24, pp. 125-139, 1992.

[46] M. Engliš, "Weighted Bergman kernels and quantization," Communications in Mathematical Physics, vol. 227, no. 2, pp. 211-241, 2002.

[47] F. Bayen, M. Flato, C. Fronsdal, A. Lichnerowicz, and D. Sternheimer, "Quantum mechanics as a deformation of classical mechanics-part I," Letters in Mathematical Physics, vol. 1, no. 6, pp. 521-530, 1977.

[48] F. Bayen, M. Flato, C. Fronsdal, A. Lichnerowicz, and D. Sternheimer, "Deformation theory and quantization-parts II and III," Annals of Physics, vol. 111, pp. 61-110, 111-115, 1978.

[49] F. A. Berezin, "Quantization," Mathematics of the USSR-Izvestiya, vol. 8, pp. 1109-1165, 1974.

[50] J. E. Moyal, “Quantum mechanics as a statistical theory,” vol. 45, pp. 99-124, 1949.

[51] H. Weyl, Gruppentheorie und Quantenmechanik, Wissenschaftliche Buchgesellschaft, Darmstadt, Germany, 2nd edition, 1977.

[52] Marc De Wilde and P. B. A. Lecomte, "Existence of star-products and of formal deformations of the Poisson Lie algebra of arbitrary symplectic manifolds," Letters in Mathematical Physics, vol. 7, no. 6, pp. 487-496, 1983.

[53] H. Omori, Y. Maeda, and A. Yoshioka, "Weyl manifolds and deformation quantization," Advances in Mathematics, vol. 85, no. 2, pp. 224-255, 1991.

[54] H. Omori, Y. Maeda, and A. Yoshioka, "Existence of closed star-products," Letters in Mathematical Physics, vol. 26, pp. 284-294, 1992.

[55] B. V. Fedosov, "Deformation quantization and asymptotic operator representation," Funktional Anal $i$ Prilozhen, vol. 25, no. 2, pp. 184-194, 1990.

[56] B. V. Fedosov, "A simple geometrical construction of deformation quantization," Journal of Differential Geometry, vol. 40, no. 2, pp. 213-238, 1994.

[57] M. Kontsevich, "Deformation quantization of Poisson manifolds," Letters in Mathematical Physics, vol. 66, no. 3, pp. 157-216, 2003.

[58] P. Deligne, “Déformations de l'algèbre des fonctions d'une variètè symplectique: comparaison entre Fedosov et De Wilde, Lecomte," Selecta Mathematica. New Series, vol. 1, no. 4, pp. 667-697, 1995.

[59] S. Gutt and J. Rawnsley, "Equivalence of star products on a symplectic manifold; an introduction to Deligne's Čech cohomology classes," Journal of Geometry and Physics, vol. 29, no. 4, pp. 347-392, 1999.

[60] M. Bertelson, M. Cahen, and S. Gutt, "Equivalence of star products," Classical and Quantum Gravity, vol. 14, no. 1, pp. A93-A107, 1997.

[61] R. Nest and B. Tsygan, "Algebraic index theorem," Communications in Mathematical Physics, vol. 172, no. 2, pp. 223-262, 1995. 
[62] R. Nest and B. Tsygan, "Algebraic index theory for families," Advances in Mathematics, vol. 113, pp. 151-205, 1995.

[63] A. V. Karabegov, "Deformation quantizations with separation of variables on a Kähler manifold," Communications in Mathematical Physics, vol. 180, no. 3, pp. 745-755, 1996.

[64] M. Bordemann and St. Waldmann, "A Fedosov star product of the Wick type for Kähler manifolds," Letters in Mathematical Physics, vol. 41, no. 3, pp. 243-253, 1997.

[65] N. Reshetikhin and L. A. Takhtajan, "Deformation quantization of Kähler manifolds," in L. D. Faddeev's Seminar on Mathematical Physics, vol. 201 of American Mathematical Society Translations: Series 2, pp. 257-276, American Mathematical Society, Providence, RI, USA, 2000.

[66] M. Schlichenmaier, "Berezin-Toeplitz quantization of compact Kähler manifolds," in Quantization, Coherent States and Poisson Structures, A. Strasburger, S. T. Ali, J.-P. Antoine, J.-P. Gazeau, and A. Odzijewicz, Eds., pp. 101-115, Polish Scientific Publisher PWN, 1998, Proceedings of the 14th Workshop on Geometric Methods in Physics (Białowieża, Poland, July 1995).

[67] M. Schlichenmaier, "Deformation quantization of compact Kähler manifolds via Berezin-Toeplitz operators," in Proceedings of the 21st International Colloquium on Group Theoretical Methods in Physics, H.-D. Doebner, P. Nattermann, and W. Scherer, Eds., pp. 369-400, World Scientific, Goslar, Germany, 1996.

[68] E. Hawkins, "The correspondence between geometric quantization and formal deformation quantization," http://arxiv.org/abs/physics/9811049.

[69] D. Borthwick, T. Paul, and A. Uribe, "Semiclassical spectral estimates for Toeplitz operators," Annales de l'Institut Fourier, vol. 48, no. 4, pp. 1189-1229, 1998.

[70] A. V. Karabegov, "Cohomological classification of deformation quantizations with separation of variables," Letters in Mathematical Physics, vol. 43, no. 4, pp. 347-357, 1998.

[71] A. V. Karabegov, "On the canonical normalization of a trace density of deformation quantization," Letters in Mathematical Physics, vol. 45, no. 3, pp. 217-228, 1998.

[72] V. Guillemin, "Some classical theorems in spectral theory revisited," in Seminar on Singularities of Solutions of Linear Partial Differential Equations, L. Hörmander, Ed., vol. 91 of Annals of Mathematics Studies, pp. 219-259, Princeton University Press, Princeton, NJ, USA, 1979.

[73] J. H. Rawnsley, "Coherent states and Kähler manifolds," The Quarterly Journal of Mathematics. Oxford. Second Series, vol. 28, no. 112, pp. 403-415, 1977.

[74] J. Rawnsley, M. Cahen, and S. Gutt, “Quantization of Kähler manifolds. I. Geometric interpretation of Berezin's quantization," Journal of Geometry and Physics, vol. 7, no. 1, pp. 45-62, 1990.

[75] M. Cahen, S. Gutt, and J. Rawnsley, "Quantization of Kähler manifolds. II," Transactions of the American Mathematical Society, vol. 337, no. 1, pp. 73-98, 1993.

[76] M. Cahen, S. Gutt, and J. Rawnsley, “Quantization of Kähler manifolds. III," Letters in Mathematical Physics, vol. 30, no. 4, pp. 291-305, 1994.

[77] M. Cahen, S. Gutt, and J. Rawnsley, "Quantization of Kähler manifolds. IV," Letters in Mathematical Physics, vol. 34, no. 2, pp. 159-168, 1995.

[78] S. Berceanu and M. Schlichenmaier, "Coherent state embeddings, polar divisors and Cauchy formulas," Journal of Geometry and Physics, vol. 34, no. 3-4, pp. 336-358, 2000.

[79] C. Moreno and P. Ortega-Navarro, "*-products on $D 1(\mathbb{C}), S^{2}$ and related spectral analysis," Letters in Mathematical Physics, vol. 7, no. 3, pp. 181-193, 1983.

[80] C. Moreno, "*-products on some Kähler manifolds," Letters in Mathematical Physics, vol. 11, no. 4, pp. 361-372, 1986.

[81] M. Engliš, "Berezin quantization and reproducing kernels on complex domains," Transactions of the American Mathematical Society, vol. 348, no. 2, pp. 411-479, 1996.

[82] M. Engliš, "Asymptotics of the Berezin transform and quantization on planar domains," Duke Mathematical Journal, vol. 79, no. 1, pp. 57-76, 1995.

[83] M. Engliš, "The asymptotics of a Laplace integral on a Kähler manifold," Journal für die Reine und Angewandte Mathematik, vol. 528, pp. 1-39, 2000.

[84] A. Unterberger and H. Upmeier, "The Berezin transform and invariant differential operators," Communications in Mathematical Physics, vol. 164, no. 3, pp. 563-597, 1994.

[85] M. Engliš and J. Peetre, "On the correspondence principle for the quantized annulus," Mathematica Scandinavica, vol. 78, no. 2, pp. 183-206, 1996.

[86] M. Schlichenmaier, "Berezin-Toeplitz quantization and Berezin transform," in Long Time Behaviour of Classical and Quantum Systems, vol. 1, pp. 271-287, World Scientific, River Edge, NJ, USA, 2001. 
[87] M. Schlichenmaier, "Berezin-Toeplitz quantization and Berezin symbols for arbitrary compact Kähler manifolds," in Proceedings of the 17th Workshop on Geometric Methods in Physics, M. Schlichenmaier, et al., Ed., pp. 45-56, Warsaw University Press, Białowieża, Poland, 1998.

[88] L. Boutet de Monvel and J. Sjöstrand, "Sur la singularité des noyaux de Bergman et de Szegö," in Journées: Équations aux Dérivées Partielles de Rennes, Asterisque, pp. 123-164, Société Mathématique de France, Paris, France, 1976.

[89] S. Zelditch, "Szegö kernels and a theorem of Tian," International Mathematics Research Notices, no. 6, pp. 317-331, 1998.

[90] G. Tian, “On a set of polarized Kähler metrics on algebraic manifolds," Journal of Differential Geometry, vol. 32, no. 1, pp. 99-130, 1990. 


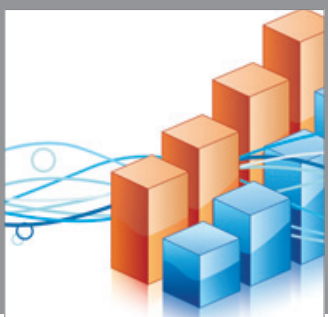

Advances in

Operations Research

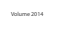

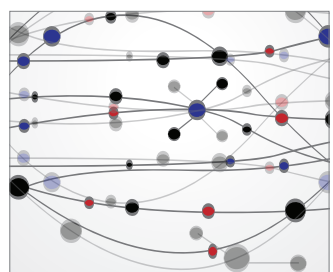

\section{The Scientific} World Journal
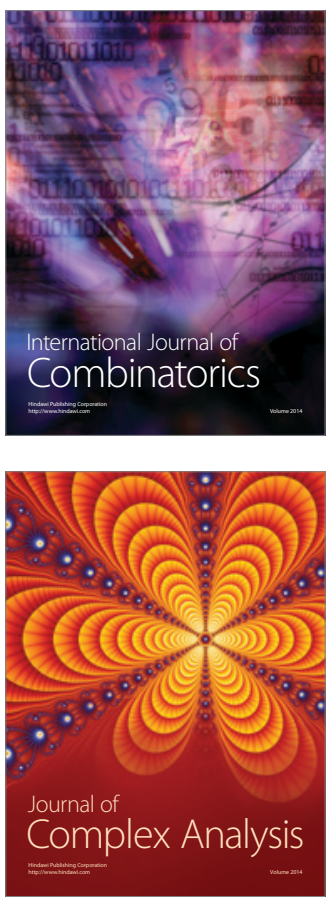

International Journal of

Mathematics and

Mathematical

Sciences
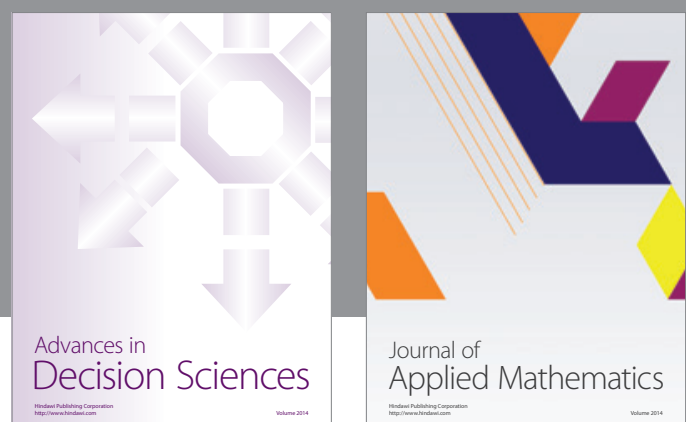

Journal of

Applied Mathematics
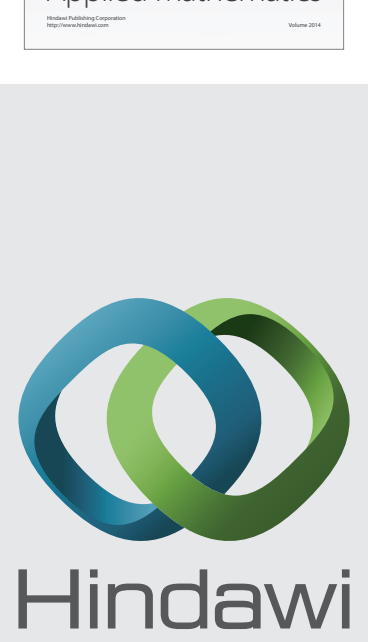

Submit your manuscripts at http://www.hindawi.com
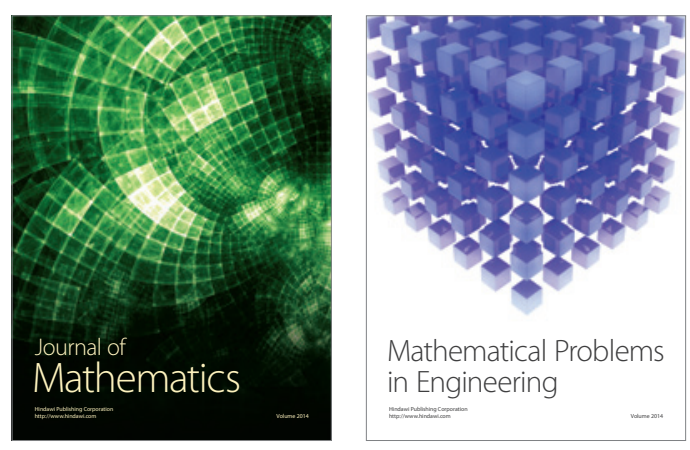

Mathematical Problems in Engineering
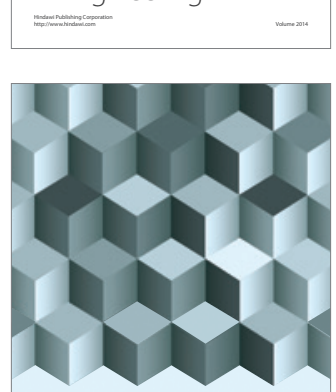

Journal of

Function Spaces
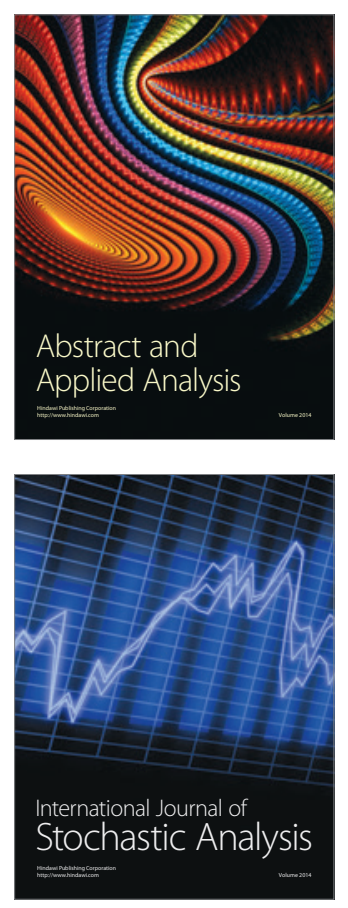

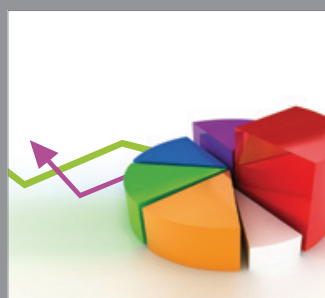

ournal of

Probability and Statistics

Promensencen
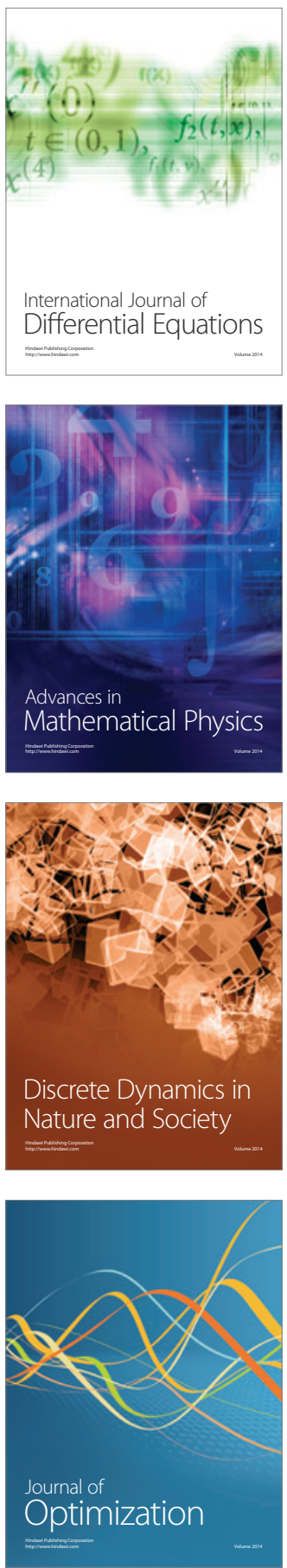